\title{
Initializing the WRF Model with Tropical Cyclone Real-Time Reports using the Ensemble
}

\section{Kalman Filter Algorithm}

${ }^{(1)}$ National Center for Hydro-Meteorological Forecasting, 8 Phao Dai Lang, Hanoi, Vietnam

${ }^{(2)}$ Department of Space and Aeronautics, University of Science and Technology of Hanoi, Vietnam

${ }^{(3)}$ Department of Earth and Atmospheric Sciences, Indiana University, Bloomington IN 47405, USA

Revised: 18 April 2017

Submitted to Pure and Applied Geophysical Science

Abbreviated title: Tropical Cyclone Ensemble Forecast

Keywords: Tropical cyclones, ensemble Kalman filter, the WRF model, tropical cyclone vital, ensemble forecasting

*Corresponding author: Chanh Kieu, Atmospheric Program, GY428A Geological Building, Department of Earth and Atmospheric Sciences, Indiana University, Bloomington, IN 47405. Tel: 812-856-5704. Email: ckieu@,indiana.edu. 


\section{Abstract}

This study presents an approach to assimilate tropical cyclone (TC) real-time reports and the

3 University of Wisconsin-Cooperative Institute for Meteorological Satellite Studies (CIMSS)

4 Atmospheric Motion Vectors (AMV) data into the Weather Research and Forecasting (WRF) model

5 for TC forecast applications. Unlike current methods in which TC real-time reports are used to either

6 generate a bogus vortex or spin-up a model initial vortex, the proposed approach ingests the TC real-

7 time reports through blending a dynamically consistent synthetic vortex structure with the CIMSS-

8 AMV data. The blended dataset is then assimilated into the WRF initial condition, using the local

9 ensemble transform Kalman filter (LETKF) algorithm. Retrospective experiments for a number of

10 TC cases in the north Western Pacific basin during 2013-2014 demonstrate that this approach could

11 effectively increase both the TC circulation and enhance the large-scale environment that the TCs are

12 embedded in. Further evaluation of track and intensity forecast errors shows that track forecasts

13 benefit more from improvement in the large-scale flow at 4-5 day lead times, whereas the intensity

14 improvement is minimal. While the difference between the track and intensity improvement could be

15 due to a specific model configuration, this result appears to be consistent with the recent reports of

16 insignificant impacts of inner-core data assimilation in operational TC models at the long range of 4-

175 days. The new approach will be most beneficial for future regional TC models that are directly

18 initialized from very high-resolution global models whose storm initial locations are sufficiently

19 accurate at the initial analysis that there is no need to carry out any artificial vortex removal or filtering 20 steps. 


\section{Introduction}

Studies of tropical cyclone (TC) initialization for real-time forecasts have shown that incipient inner-core structure of TCs can have substantial impacts on TC initial adjustment and subsequent

4

track and intensity development (see, e.g., Kurihara et al. 1993; Wang 1998; Hendricks et al. 2011, 2013; Nguyen and Chen 2011). Given such importance of the TC initial structure, numerous effort has been carried out to improve the initial representation of TC inner-core structure in operational TC models. General approaches to improve the TC initial inner-core can be roughly divided into three categories including i) TC bogussing methods that replace an initially weak cyclonic perturbation by a bogus vortex with a prescribed intensity (Ross and Kurihara 1992; Kurihara et al. 1993; Liu et al. 2006), ii) dynamical vortex initialization methods that use a numerical model to spin-up an initial vortex (Nguyen and Chen 2011, 2014; Cha and Wang 2013; Hendricks et al. 2013), and iii) assimilation methods that directly ingest TC observations to enhance the TC initial condition (Zhang et al. 2006; 2011; Xu et al. 2013; Wang et al. 2014; Holt et al. 2015). The first approach requires a bogussed vortex that is frequently generated by using either TC empirical balanced profiles (e.g., Fujita 1952; Holland 1980) or a given vortex library (Ross and Kurihara 1992; Kurihara et al. 1993; Liu et al. 2006), whereas the assimilation approaches could directly ingest different sources of observations into initial analysis without any artificial vortex filtering processes.

While vortex bogussing methods can produce a model initial vortex that better fits with the observed intensity in TC real-time reports $\left(\mathrm{TCR}^{1}\right)$, the practice of $\mathrm{TC}$ initialization in operational models shows that a bogussed vortex often introduces unexpected initial adjustment and results in

\footnotetext{
${ }^{1}$ The TC real-time reports are often referred to as TC vital record in operational environment. In the north Western Pacific basin, TC vital records and advisories are provided in real-time by the US Joint Typhoon Warning Center (JTWC), which include basic information of the storm center position, the maximum surface wind, the minimum sea level pressure, and wind radii at different quadrants.
} 
1 strong spin-down or spin-up of the model storm during the first 6-12 hours before the model storm

2 can adapt to the environment (Hendricks et al. 2013; Nguyen and Chen 2011; Tong et al. 2014, 2015).

3 Recent implementation of real-time assimilation of targeted observations revealed further that the TC

4 bogussing technique is not always compatible with the assimilation of the inner-core observation

5 (Tallapragada et al. 2014; Tong et al. 2014; Pu et al. 2016). Specifically, the TC bogussing process

6 attempts to generate a new vortex that best fits to observed TC intensity at the surface, whereas the

7 inner-core assimilation tends to modify the TC structure at all levels, which can result in a TC initial

8 intensity different from the best track observation. Such a conflict between the vortex bogussing and

9 the inner-core assimilation results in reduced TC intensity forecast skill, thus posing a real challenge

10 to the current operational TC forecasts (Tallapragada et al. 2014). Despite this inherent compatible

11 issue, vortex bogussing is still used as a pre-conditioning step for practical purposes to prepare a better

12 background covariance for subsequent inner-core data assimilation.

recently presented a different approach, the so-called dynamical initialization, to alleviate the initial unbalanced adjustment. The essence of this dynamical initialization is to use the model internal dynamics to spin-up a weak vortex to match with observed intensity via successive iterations and

17 relaxation. By using the maximum surface wind and the minimum sea level pressure as the dynamical constraints, Nguyen and Chen $(2011,2014)$ and Cha and Wang (2013) demonstrated that the spin-up

19 time of any TC vortex can be significantly reduced with the use of short-range forecast cycles, thus

20 making this method more suitable for practical purposes. Although the dynamical initialization could

21 take advantage of the model to avoid the initial spin-up or spin-down, the main issue with this

22 approach is the long iteration required for the model spin-up, especially when initial discrepancies in

23 the TC intensity between global analysis and observed intensity are sufficiently large, making it 
1 impractical for operational purposes in extreme situations. Also, there is no guarantee that models

2 will always spin-up any initial vortex, and in some cases models may unexpectedly spin-down an

3 initial storm rather than amplifying it.

Issues of complicated bogussing processes and the related artificial numerical effects in the

5 TC initialization have been addressed differently with recent advances in data assimilation schemes

6 (Zou and Xiao 2000, Pu and Braun 2001; Xiao and Wang 2000; Zhang et al. 2011). In fact, current

7 availability of the real-time flight reconnaissance data allows the direct assimilation of tail-Doppler

8 radar observations into the global analysis without the need of vortex bogussing (see, e.g., Zhang and

9 Weng 2015; Aberson et al. 2015). An important advantage of the ensemble Kalman filter (EnKF)

10 method as compared to variational assimilating schemes is that the background covariance matrix is

11 allowed to vary in time, thus adapting better with fast-evolving dynamical systems such as hurricanes

12 or meso-scale convective systems (Snyder and Zhang 2003).

Given recent demonstrated capability of the Local Ensemble Transform Kalman Fitter

14 (LETKF) algorithm in TC forecasts (see, e.g., Miyoshi and Kunii 2012; Kieu et al. 2012, 2014;

15 Cecelski et al. 2014; Holt et al. 2015), this study will examine the effectiveness of the LETKF

16 algorithm in assimilating the TCR information into the WRF-ARW model for real-time TC forecasts.

17 Specifically, an innovative approach based on the real-time TCR-synthetic observation that does not

18 require the storm relocation or a bogus vortex will be presented. In addition to the real-time TCR

19 information, the atmospheric motion vector (AMV) data provided by the Cooperative Institute for

20 Meteorological Satellite Studies (CIMSS) of the University of Wisconsin will be also assimilated to

21 improve the large-scale environment that controls the TC steering flows, which could have significant

22 effects on the TC track forecasts (Velden et. al. 1992; Kieu et al. 2012). 
The remainder of the paper is organized as follows. In the next section, methodology for

2 generating the TC synthetic vortex information from the real-time TCR and the ensemble assimilation

3 scheme will be presented. Section 3 describes the model experiments and ensemble designs. Section

44 discusses impacts of assimilation of the TCR-synthetic profiles and the related track and intensity

5 forecasts, and some concluding remarks are given in the final section.

\section{2. Methodology}

a. Construction of TC-synthetic profile

To help enhance representation of TC circulation in the initial global analysis without filtering

9 and inserting a bogus vortex, a synthetic observation approach based on real-time TCRs is proposed

10 in this study. Our approach to generate the synthetic vortex information about the axisymmetric

11 component of a TC vortex is to first construct a dynamically consistent wind profile that requires

12 basic parameters currently available in real-time TC vitals. While there are several different empirical

13 and analytical functional forms for the TC vertical structure, we choose a vertical profile for the

14 tangential wind proposed by Kieu and Zhang (2009), which is derived from an analytical model that

15 ensures the dynamical consistency among the mass and wind fields. For the radial distribution for the

16 tangential wind, the wind profile similar to that in Wang (1998) is adopted, which is based on several

17 input parameters including the radius of maximum wind (RMW), the maximum 10-m sustained wind

18 (VMAX), and the radius of the outermost closed isobar. Combination of these vertical and horizontal

19 profiles results in a tangential wind structure in the $\sigma$ vertical coordinate as follows

$$
V(r, \sigma)=V_{m} \frac{r}{r_{m}} \sin \left(\frac{\pi \sigma}{2}\right)^{1-\delta} \cos \left(\frac{\pi \sigma}{2}\right)^{\delta} \exp \left\{\frac{1}{b}\left[1-\left(\frac{r}{r_{m}}\right)^{b}\right]\right\}
$$

21 where $V_{m}$ is the VMAX, $r_{m}$ is the RMW, $r$ is the radius from the vortex center, $b$ is a parameter that 22 determines the horizontal shape of the wind profile, which is set equal to 0.7 by default, and $\delta \in$ 
$1[0,1]$ is a free parameter determining the height of the VMAX relative to the surface (i.e., $\delta=1$

2 corresponds to the maximum wind at the surface, whereas $\delta=0$ corresponds to the maximum wind 3 at the middle level, see Kieu et al. 2014).

With the tangential wind distribution given by Eq. (1), the three-dimensional distribution of geopotential $\Phi$ is then calculated by iteratively solving the nonlinear balance equation of the form (see Holton 2004; Kieu and Zhang 2010).

$$
\nabla^{2} \Phi=\nabla \cdot(f \nabla \psi)-J\left(\frac{\partial \psi}{\partial x}, \frac{\partial \psi}{\partial y}\right)
$$

where $f$ is the Coriolis parameter, $\psi$ is the horizontal streamfunction, $J$ is the Jacobian operator, and $\nabla$ denotes the horizontal gradient $(\partial / \partial x, \partial / \partial y)$ on the $(x, y)$ coordinates. In the final step, the balanced temperature profile is obtained by using the hydrostatic equation based on the balanced geopotential distribution as obtained from Eq. (2). For this type of a vortex structure, the axisymmetric component of TCs is thus fully constructed from several parameters available in TCRs. By assimilating this synthetic structure into the storm location, the axisymmetric component of the TC circulation can be enhanced without the need of artificially removing or inserting a bogus structure into the initial Global Forecasting System (GFS) analysis. It should be mentioned that although a background covariance matrix could encode some general balanced relationships that constrain the model wind and pressure distributions, the above construction of the TC-synthetic based on the balanced Eq. (2) is still needed so that the balance in the input synthetic data will be maximally retained. Without proper constrains of the synthetic variables, the analysis may be largely distorted, even if the background covariance matrix maintains its inherent balance.

For practical applications, note that the synthetic observation constructed from TCRs contains information related only to the storm-scale characteristics. Thus, the synthetic structure may have 
minimum impact to large-scale environment. As a step to further improve environmental conditions

2 along with the storm-scale enhancement, the CIMSS-AMV is utilized. A common approach to

3 assimilation of multiple data sources is to carry out the assimilation sequentially for each type of

4 observations (see, e.g., Tallapragada et al. 2014; Pu et al. 2016). Our proposed approach for the TC

5 initialization problem is, however, to combine both the AMV and the TCR-based synthetic vortex

6 structure into a single data tank before assimilation. There may have some data compatibility issue in

7 this data blending approach when several different data sources are used, but this issue can be

8 alleviated by assigning different observational errors to different sources so that the weight on

9 different data sources in the interference area can be tuned. In our experiments, we notice that the

10 AMV data covers mostly upper levels (above $300 \mathrm{hPa}$ ) and in the outer-core region, whereas the

11 TCR-based synthetic structure is designed only for the storm inner-core region from the surface to

$12200 \mathrm{hPa}$. As such, data compatibility is not a severe issue in this study.

\section{b. LETKF algorithm}

Various studies and experiments with different variants of the EnKF for a wide range of problems have demonstrated the capability of the EnKF in many practical mesoscale applications. Specifically, Miyoshi and Kunii (2012), Kieu et al. (2012, 2013), Cecelski et al. (2014), Holt et al. (2015) showed that the LETKF algorithm is able to effectively assimilate different sources of observational information for TC forecasts. Unlike other versions of the EnKF algorithm, LETKF performs analysis locally in an ensemble space that is independent at different grid points (Ott et al. 2002, 2004; Hong and Kalnay 2010; Hunt et al. 2007). This particular locality feature of the LETKF algorithm allows efficient parallelism design, thus reducing computational cost and making it suitable to many different applications (e.g., Hunt et al. 2004; Kang et al. 2011; Szunyogh et al. 2008; Yang et al. 2009; Liu et al. 2008; Miyoshi and Kunii 2012; Kieu et al. 2012, 2014; Holt et al. 2015). In the LETKF method, the forecast error uncertainties are estimated by using the background ensemble 
matrix as a transformation operator from the model space spanned by grid points within a given local

2 patch to the ensemble space spanned by the ensemble members. The essence of the LETKF algorithm

3 is to minimize a local cost function in the ensemble space so that the final analysis ensemble $x^{a}$ can

4 be updated as follows

$$
x^{a(i)}=\bar{x}^{b}+X^{b}\left\{\bar{w}^{a}+\left[(k-1) \hat{P}^{a}\right]_{i}^{\frac{1}{2}}\right\}
$$

6 where $\hat{P}^{a}$ is the analysis error covariance matrix, $X^{b}=\left\{x^{b(1)}-\bar{x}^{b}, x^{b(2)}-\bar{x}^{b}, \ldots, x^{b(k)}-\bar{x}^{b}\right\}$ is

7 the ensemble of background perturbations corresponding to an ensemble $\left\{x^{b(i)}: i=1,2 \ldots, k\right\}, k$ is

8 the number of ensemble members, $\bar{x}^{b}=\frac{1}{k} \sum_{i=1}^{k} x^{b(i)}$ is the background ensemble mean, and $\bar{w}^{a}$ is

9 the mean analysis state in the ensemble space (see Hunt et al. 2007). To further take into account the

10 model errors, one can introduce a multiplicative factor to $\hat{P}^{a}$. More detailed derivation and handling

11 of general nonlinear and asynchronous observations in the LETKF can be found in Hunt et al. (2007).

To reduce spurious correlations in the LETKF algorithm, a local volume in the model space

13 of the size $(5 \times 5 \times 3)$ grid points in the $(\mathrm{x}, \mathrm{y}, \sigma)$ direction is used. This choice of the local volume is

14 sufficient to ensure that both AMV and synthetic observations within the local volume surrounding a

15 given grid point can be used to effectively update the grid point (see, e.g., Kieu et al. 2012, 2013).

16 Note that a too large local volume will introduce an overfitting of the observation when the number

17 of observations is larger than the local dimension spanned by the local volume, thus reducing the

18 benefit of the observational data (see, e.g., Tsyrulnikov 2009). In contrast, a too small volume will

19 have so few observational data to update the model grid points.

The performance of the LETKF algorithm for TC forecasting applications in this study is enhanced further with the multiple-physics ensemble and a fixed multiplicative inflation factor of 1.1 
1 to take into account potential model errors. As discussed in Kieu et al. (2013), the model errors in the

2 LETKF system are sensitive to the multiple-physics option, which accounts for a larger track and

3 intensity ensemble spread than the spread associated with the multiplicative inflation alone. More

4 details about implementation of the multiple-physics ensemble for the WRF-LETKF system used in

5 this study and the sensitivity of the LETKF ensemble spread on the multiplicative inflation factors

6 can be found in Kieu et al. (2012, 2013).

7

8

\section{Experiment design}

\section{a. Model description}

In this study, the Weather Research and Forecasting model (WRF-ARW, version 3.2, Skamarock et al. 2008) with two-way interactive, movable, multi-nested grid is chosen for all ensemble vortex initialization experiments. The model is configured with three nested-grid domains consisting of $151 \times 151,100 \times 100$, and $151 \times 151$ grid points in the $(x, y)$ dimensions whose horizontal resolutions are $36 \mathrm{~km}, 12 \mathrm{~km}$, and $4-\mathrm{km}$, respectively. The outermost domain is fixed and covers an area of $\sim 5500 \mathrm{~km} \times 5500 \mathrm{~km}$ with the domain center at $120^{\circ} \mathrm{E}, 25^{0} \mathrm{~N}$, whereas the intermediate domain $(12-\mathrm{km})$ and the innermost domain $(4-\mathrm{km})$ are set to automatically follow TC centers using a build-in tracking algorithm in the WRF-ARW model. The model is integrated for 5 days with a time step of $120 s$ and the lateral boundary conditions updated every 6 hours, using the National Centers for Environmental Prediction (NCEP) Global Forecasting System (GFS) real-time forecasts at resolution of $0.5^{0} \times 0.5^{0}$. All three domains share the same 31 vertical $\sigma$ levels with the model top set at $50 \mathrm{hPa}$.

For the purpose of multiple-physics ensemble design, a set of physical parameterizations used in all ensemble experiments include (a) the modified Kain-Fritsch and Betts-Miller-Janjic cumulus parameterization schemes; (b) the Yonsei University and Mellor-Yamada-Janjic planetary boundary 
1 layer (PBL) parameterization with the Monin-Obukhov surface layer scheme; (c) the Rapid Radiative

2 Transfer Model scheme for longwave radiation and the Goddard and Dudhia schemes for the

3 shortwave radiation; and (d) the Kessler, the Lin, the Eta (Ferrier), the WSM3, the WSM5, and the

4 WSM6 schemes for the cloud microphysics (see Skamarock et al. 2008). There is no cumulus

5 parameterization for the 4-km resolution domain. For multiple-physics ensemble experiments, each

6 specific combination of physical parameterization schemes is assigned to one ensemble member in a

7 sequence such that the ensemble will have as many combinations as the model allows. Given 2

8 cumulus parameterization schemes, 2 PBL schemes, 2 radiation schemes, and 6 microphysics

9 schemes, there are totally 48 different combinations. However, a large number of combinations are

10 not compatible due to model internal physics requirements, and so only 21 different combinations out

11 of total 48 possibilities are alternated in our multiple-physics ensemble. More detailed description of

12 the WRF-ARW model and the constraints in combination of physical parameterization schemes can

13 be found in Skamarock et al. (2008).

\section{b. Ensemble experiments}

As an illustration of how the blending of the TCR-based profiles and the CIMSS/AMV data

helps improve TC forecasts with the LETKF algorithm, all strong cycles of the six Super Typhoons and Typhoon cases over the northwestern Pacific basin are experimented in this study. Table 2 lists the cycles of strong intensity for each storm, which are selected to capture the most effectiveness of 19 the TCR-based synthetic inner-core assimilation.

For each TC case, three sets of ensemble experiments are carried out to examine effects of the

21 TCR-based synthetic observation, the CIMSS-AMV data, as well as the effectiveness of the LETKF

22 algorithm. All ensemble experiments are configured with a fixed number of 21 ensemble members.

23 In the first control ensemble experiment (CTRL), a set of initial conditions is generated by adding 24 random Gaussian noise with standard deviations of $1.4 \mathrm{~m} \mathrm{~s}^{-1}$ for wind, $1.4 \mathrm{~K}$ for temperature, and 3 
$1 \times 10^{-3} \mathrm{~kg} \mathrm{~kg}^{-1}$ for specific humidity to GFS analyses without assimilation of any TC synthetic profiles

2 or CIMSS-AMV data. Similar to the configuration used in Kieu et al. (2013), multiple physical

3 parameterizations with different cumulus parameterizations, cloud microphysics and longwave

4 radiation schemes are also used in the CTRL experiments to increase model representation of large-

5 scale uncertainties (a list of parameterization schemes are provided in Table 1). These CTRL

6 experiments serve as references upon which the effectiveness of assimilations of the CIMSS-AMV

7 data and TCR-based synthetic profiles can be evaluated.

8 In a second set of experiments (DAMV), the CIMSS-AMV data is assimilated into GFS

9 analyses, but no TCR-based synthetic profiles are employed. The DAMV experiments are to

10 investigate the impacts of the CIMSS-AMV data as compared to the CTRL experiments. As shown

11 in Kieu et al. (2013), the CIMSS-AMV could help enhance large-scale steering flows, thus improving

12 TC track and subsequently intensity forecasts. To sample model errors that a simple multiplicative

13 inflation technique could not address, the same combinations of the model physical parameterizations

14 as in the CTRL experiments are applied to all ensemble members. Differences between the DAMV

15 and CTRL experiments will demonstrate how much the large-scale information from the CIMSS-

16 AMV data can affect the large-scale steering flows and upper-level circulations of TCs.

In the last set of experiments (DABV), a similar ensemble of 21 members with identical model

18 configurations as in the CTRL and DAMV experiments are used, but both the AMV observations and

19 the TCR-based synthetic observations are blended before being assimilated to GFS analyses. Note

20 again that unlike the traditional 2-step approach in which bogus vortex initialization is carried out

21 first and the inner-core assimilation is subsequently followed, our proposed approach is to

22 simultaneously blend both the AMV data and the TC synthetic observations into a single database

23 before conducting any assimilation. Outcomes from this DABV experiment will show how 
1 enhancement of the TC inner-core structure based on TCRs could impact intensity forecasts as

2 compared to CTRL forecasts or assimilation of the AMV data only. Table 3 summarizes all

3 configurations and setup for the three experiments. Because the inner-core assimilation using the

4 synthetic data alone is generally insufficient to help improve track forecasts (see, e.g., Tong et al.

5 2014), the blending of the TCR-synthetic data with the AMV data is therefore necessary to prevent

6 the degrade of track forecasts that may ultimately affect the TC intensity at the long ranges. As such,

7 no separate experiments with pure assimilation of the TCR-based synthetic observations are

8 conducted in this study.

9 Due to the requirement of initializing cold-start ensembles in all practical applications of TC

10 forecasts with regional models, we follow the same approach as in Zhang et al. (2006) and initialize

11 cold-start background ensembles by adding a random noise with standard deviations of $1.4 \mathrm{~m} \mathrm{~s}^{-1}$ for

12 zonal wind components, $1.4 \mathrm{~K}$ for temperature, and $2 \times 10^{-3} \mathrm{~kg} \mathrm{~kg}^{-1}$ for specific humidity into the

13 NCEP/GFS operational analysis, which are consistent with the default background covariance errors

14 set in the WRF three-dimensional variational data assimilation (WRFDA) system. These random

15 perturbations are maximum as at the model lowest level, and change with height to account for

16 variation of the background error covariance matrix at different vertical levels. Because current GFS

17 initial analyses capture relatively well storm intensity during early stages of TC development, the

18 blending of the synthetic structure with the AMV data is activated only when the initial intensity

19 difference between the GFS analysis and the observed VMAX is larger than a prescribed threshold,

20 which is set $30 \mathrm{~m} \mathrm{~s}^{-1}$ in our current system. For storms with initially weaker intensity, by default only

21 the AMV data is used in our current WRF-LETKF system.

22 c. Data

23 As an attempt to demonstrate our proposed approach of blending the TCR-based synthetic

24 vortex profile and the AMV data for real-data TC forecasts, six TC cases in 2013 and 2014 over the 
1 north Western Pacific basin are chosen, including Super Typhoon Usagi from 0000 UTC 18-19

2 September 2013, Typhoon Krosa from 0000 UTC 30-31 September 2013, Super Typhoon Nari from

30000 UTC 10-11 October 2013, Super Typhoon Neoguri from 0000 UTC 05-06 July 2014, Super

4 Typhoon Rammasun on 0000 UTC and 1200 UTC 15 July 2014 and Super Typhoon Vongfong on

50000 UTC and 1200 UTC 06 October 2014 (Fig. 1). The model initial and lateral boundary conditions

6 for these cases are taken from the GFS real-time forecasts at a horizontal resolution of $0.5^{0} \times 0.5^{0}$,

7 with boundary conditions updated every 6 hours.

For TCRs, the Tropical Cyclone Vital Statistics Records from US Joint Typhoon Warning

9 Center (JTWC) are used to provide all necessary inputs for constructing synthetic bogus structures as

10 described in the previous section. JTWC's real-time TCRs include key information such as the TC

11 identification number and basin, latitudes and longitudes of TC centers, the VMAX, the minimum

12 sea level pressure, the outermost isobaric radius, the RMW, and the radii of the $34 \operatorname{knot}\left(\sim 17 \mathrm{~m} \mathrm{~s}^{-1}\right)$,

$1350 \operatorname{knot}\left(\sim 20 \mathrm{~m} \mathrm{~s}^{-1}\right)$, and $64 \operatorname{knot}\left(\sim 32 \mathrm{~m} \mathrm{~s}^{-1}\right)$ wind speed from the TC center. Given these parameters,

14 a vortex structure consisting of the tangential wind, the temperature perturbation, and the geopotential

15 height perturbation is then constructed at every analysis cycle using Eqs. (1) and (2). These profiles

16 are then discretized at the same horizontal and vertical resolution of the outermost domain, and then

17 interpolated to the innermost domain at the resolution of $4 \mathrm{~km}$ before assimilated. For each of the data

18 point, statistics of observational errors similar to those given by the WRFDA background error

19 covariance are used. Detailed explanation and format of the TCR reports can be found in

20 http://www.emc.ncep.noaa.gov/mmb/data processing/tcvitals description.htm, and the real-time TC

21 record for any specific cycle can be downloaded from the NCEP product server at

$22 \mathrm{ftp} / / / \mathrm{ftp}$. ncep.noaa.gov/pub/data/nccf/com/gfs/prod/, once a TC emerges. 
2 dataset retrieved and post-processed by CIMSS-University of Wisconsin is employed in this study.

3 This CIMSS-AMV data is quality-controlled and calibrated such that each data point is checked for

4 the overall consistency with the surrounding data using the quality indicator technique (Berger et al.

5 2007). In all experiments, only the Western Pacific basin dataset relevant to the TC cases in Table 2

6 is used, which is available at http://tropic.ssec.wisc.edu/archive/data/NWPacific. Even though the

7 GFS real-time products include all available satellite information in its global variational assimilation

8 system at each analysis time, re-assimilating the CIMSS-AMV in this case can still help enhance

9 detailed mesoscale features at higher resolution as well as restore any loss of information during the

10 interpolation from the GFS coarser grid to nested grids in limited-area models (Kieu et al. 2012).

After blending the TCR-synthetic data with the AMV data into a single data tank, both the

12 TCR-synthetic profiles and AMV observations are subject to a quality control of the WRFDA

13 component before being assimilated by the LETKF system. For the sake of our WRF-LETKF system

14 design with a current limited data transfer speed at the Vietnam National Center for Hydro-

15 Meteorological Forecasting, separate lateral boundary conditions are generated for each ensemble member by using a utility in the WRFDA system in all three experiments, instead of inheriting from

17 GFS global ensemble forecasts. By doing this, each ensemble member possesses its own lateral

18 boundary that is dynamically consistent with its own analysis. More information about the WRFDA

19 system can be found in Barker et al. (2012). For verification of the track and intensity forecast errors,

20 all experiments are verified against the best track data provided also by the JWTC. Information and

21 the latest dataset for the observed TC post-analysis (hereinafter referred to as the best track or

22 observation) can be found at the following link https://metoc.ndbc.noaa.gov/JTWC/.

\section{Results}


To examine first the impacts of assimilating TCR-based synthetic data on the storm initial

structure, Figs. 2 and 3 compare horizontal cross sections of the mean analysis innovations (or increments) and the synthetic innovations for both the zonal and meridional wind components that are obtained from the DABV experiments with Typhoons Usagi and Krosa. Here, the mean analysis innovations are defined as $\delta \chi_{a}=\bar{\chi}_{a}-\bar{\chi}_{b}$, and the synthetic innovations are $\delta \chi_{o}=\chi_{o}-\bar{\chi}_{b}$, where $\chi$ denotes either the zonal or meridional wind component, the overbar denotes the ensemble average, and the subscripts $(o, b, a)$ denote synthetic observations, forecast background, and analysis respectively.

One notices in Figs. 2-3 that the TCR-synthetic innovations $\delta \chi_{o}$ indeed exhibit a consistent wind structure with strong cyclonic wind components at all three levels 850, 500, and $200 \mathrm{hPa}$. Such large cyclonic wind innovations are expected because storm initial intensity in the GFS background is generally much weaker than the observed intensity after a TC becomes sufficiently strong (cf. Fig. 1b). Direct comparison of the VMAX from the GFS analysis and the observed intensity for these two cycles of Krosa and Usagi shows that the GFS vortex is on average $12-15 \mathrm{~m} \mathrm{~s}^{-1}$ weaker than what reported in TCRs, thus accounting for the large synthetic innovations seen in Fig. 2. In addition to the difference in storm intensity, the large synthetic innovations are also due to the fact that the GFS storm centers are not the same as those reported in TCRs. This type of large wind innovations associated with difference between the GFS storm centers and the observed storm centers is inherently related to the characteristics of TC circulation with a well-defined eye region where the wind field vanishes at the storm center. Large innovations related to such mismatched storm centers are particularly apparent for strong storms whose slight difference in the storm center could produce a very large dipole of positive/negative wind increments. Such an impact of different storm centers is 
1 very substantial, even when the GFS background vortex has the same intensity as that reported in

2 TCRs, and accounts for large synthetic wind innovations as shown in Figs. 2-3.

Given the distribution of the synthetic wind innovations with a tripole innovation structure

4 (i.e., the distribution of alternating positive/negative/positive increments) for both the zonal and

5 meridional winds around the vortex center as seen in Figs. 2 and 3, it is of importance to notice that

6 the LETKF analysis innovations could exhibit similar patterns and magnitudes at all levels.

7 Specifically, consistent enhancement of the TC inner-core circulation with two peaks of analysis wind

8 innovations $>15 \mathrm{~m} \mathrm{~s}^{-1}$ (negative to the north and positive to the south of the vortex center) is observed

9 in both cases, indicating the benefit of the synthetic innovations in correcting both the storm intensity

10 and the initial location. The peaks of the analysis wind innovations are most apparent from $850 \mathrm{hPa}$

11 to $500 \mathrm{hPa}$ levels, but less clear above $200 \mathrm{hPa}$ due to the rapid decrease of the synthetic wind with

12 height. Of further interest is that the analysis innovations could capture strong asymmetry of the zonal

13 wind due to existence of strong easterly mean flows in the northwestern Pacific basin (cf. Figs. 2a,

14 d). For example, the assimilation of synthetic observations could allow large negative u-wind analysis

15 innovations to the west of Usagi's center with values $\sim 15-20 \mathrm{~m} \mathrm{~s}^{-1}$ as compared to much weaker

16 analysis innovations of $\sim 10 \mathrm{~m} \mathrm{~s}^{-1}$ to the east of Usagi's center, similar to the synthetic innovations.

17 In this regard, the TCR-based synthetic profile data can enhance the TC intensity and help re-locate

18 the GFS initial storm center as expected.

Although the consistent tripole structures between the analysis and observed wind increments

20 indicate that the LETKF algorithm is capable of enhancing the TC inner-core structure, it is worth

21 pointing out that the centers of the analysis wind innovations do not completely coincide with the

22 synthetic innovations at higher levels (see, e.g., Fig. 2b or $\mathbf{3 b}$ ). This is because real storms are often

23 tilted with height that the TCR-based synthetic data given by Eq. (1) cannot capture. Such influence 
1 of the vortex tilting on the storm vertical structure and the extent to which the TCR-based synthetic

2 information can be assimilated in the GFS analysis is seen most clearly in the vertical cross sections

3 through the storm center (Fig. 4). Because the GFS background contains information about the vortex

4 titling structure at the analysis time, the analysis wind innovations appear to include this titling

5 information even after being enhanced with the axisymmetric structure from the synthetic data, thus

6 accounting for the slight difference between the synthetic wind innovations and the analysis

7 innovations above $500 \mathrm{hPa}$. In addition to the tilting effect, note also that the storm size could play a

8 role in assimilating the synthetic information. Indeed, figures 3-4 show that the analysis innovations

9 are more consistent with synthetic innovations for the case of Typhoon Usagi, whereas the case of

10 Typhoon Krosa tends to have a larger discrepancy between the synthetic and the analysis innovations

11 due to Krosa's smaller inner-core size $\left(\sim 3^{0} \times 3^{0}\right.$ as compared to $\sim 5^{0} \times 5^{0}$ as for the cases of Usagi)

12 and weaker intensity.

13 Another significant benefit of assimilation of the TCR-synthetic profiles is that even though

14 the reported VMAX is the 10-m wind near the surface, a synthetic profile constructed from the

15 VMAX and other surface information could allow for the spread of the surface information to higher

16 levels (cf. Fig. 4). This spread of the surface information is necessary so that the synthetic

17 observations provide a coherent vortex structure throughout the troposphere rather than confined near

18 the surface. In this regard, the augmented axisymmetric vortex structure within the troposphere while

19 retaining the tilting structure in the GFS background is an important advantage of the TCR-based

20 synthetic data. Such an advantage reflects our purpose of enhancing TC axisymmetric components

21 but on the other hand retaining the GFS asymmetric structure, thus demonstrating the effectiveness

22 of the TCR-based synthetic information in augmenting the TC circulation as designed. 

the mass, the wind, and the moisture fields is also clearly seen in Figs. 5-6, which show the analysis

3

4

innovations of the potential temperature perturbation and specific humidity. Consistent with the wind innovations seen in Figs 3-4, the temperature analysis innovations show existence of a warm core anomaly at all levels in the inner-core region with a magnitude as large as $25 \mathrm{~K}$ for the case of Usagi at its peaked intensity, and similarly for the case of Krosa albeit the magnitude of the warm anomaly is weaker. Corresponding to this warm core enhancement is the increase in the moisture content at the GFS storm center and a decrease of the moisture in the observed storm center (i.e., the development of a dry eye region at the observed location as seen in Fig. 6). Such a shift in the dry eye region from the GFS vortex center to the observed vortex center indicates that the LETKF is able to correct not only the wind field but also the temperature and the moisture field, based only on the synthetic profiles.

\section{b. Impact of the AMV data}

Given that the TC inner-core structure and intensity can be enhanced by assimilating TCRsynthetic observation as presented in the previous section, the next question is how the inclusion of the CIMSS-AMV data could help further improve large-scale ambient conditions that the TCR synthetic data could not achieve. To this end, Fig. 7 shows horizontal cross sections of the analysis innovations of wind vectors at $500 \mathrm{hPa}$ and $200 \mathrm{hPa}$ levels obtained in the DAMV experiments for the two cycles shown in Figs. 4-6. Consistent with characteristics of the AMV data, a much higher density of the AMV data points is found at upper levels above $300 \mathrm{hPa}$, whereas data points at lower levels are mainly in the peripheral area of the storm central region. As such, assimilation of the AMV data alone would not capture much information about either the storm inner-core structure or the storm intensity at lower levels. As displayed in Fig. 7, the analysis wind vector innovations show a broader cyclonic circulation at $200 \mathrm{hPa}$ similar to the observed vector innovations. Such increments 
1 of the cyclonic wind at upper levels confirm that the anticyclonic circulation associated with TC

2 outflows in the GFS analysis is overall weaker than that observed by the AMVs, which accords well

3 with a weaker intensity at the surface.

While the vortex structure inside a radius of $\sim 100-200 \mathrm{~km}$ is not directly enhanced by the

5 AMV observation below $300 \mathrm{hPa}$, we should mention that the AMV data does help improve ambient environment in the vicinity of TCs. In particular, strengthened outflow at higher levels can help a model storm reduce initial spin-up time even without changes to the storm inner-core structure at

8 lower levels. Furthermore, the correction of TC environmental flows in the peripheral area tends to 9 improve subsequent storm motion, which provides correct environmental conditions for TC development. In fact, a study by Kieu et al. (2012) demonstrated that enhancing environmental flows

11 could reduce track forecast biases for the case of Typhoon Megi (2010), thus significantly improving

12 the storm intensity after several forecast cycles.

With the capability of enhancing the storm-scale structure by the TCR-based synthetic

14 information and augmenting large-scale flows by the AMV data, it is thus natural to consider next a

15 combination of these data sources to improve both the storm-scale structure and the environmental

16 flow. Similar to Fig. 7, Fig. 8 shows comparison of the observed and the analysis innovations for

17 wind vectors in the DABV experiments. As expected, the cyclonic vector wind innovations associated

18 with the TCR-synthetic data show consistent enhancement in the storm central region at lower levels,

19 whereas the upper level circulation correctly captures the stronger anticyclonic outflow as dictated by

20 the AMV data similar to Fig. 7. The consistency between the LETKF analysis innovations and the 21 synthetic/AMV innovations demonstrates that our approach of blending the TCR synthetic data and

22 the AMV data can effectively improve the representation of TC circulations both in the inner-core 23 region and the large-scale environment with the LETKF algorithm. 


\section{c. Track and intensity forecast performance}

To finally examine the impacts of the TCR-synthetic data and the AMV data on track and intensity forecasts, Figs. $\mathbf{9}$ and $\mathbf{1 0}$ show illustrations of two ensemble track and intensity forecasts obtained from the CTRL, DAMV, and DABV experiments, respectively. For the case of Typhoon Krosa, the CTRL track forecast exhibits strong northward drift of the storm motion after just 18 hours into integration, and subsequently possesses a large southward bias as compared to the best track (Fig. 9b). The northward drift of the CTRL track is reduced in subsequent cycles, but the bias to the south is still significant after approaching $22^{\circ} \mathrm{N}$, thus resulting in overall large track error after 3 days into integration. In the case of Usagi, the CTRL track bias is small for all ensemble members during the first 48 hours, but it turns southward after 3 days and results also in large track errors at the 4-5 day lead time. Note that all CTRL ensemble members share similar southward track bias in both cycles of Krosa and Usagi, thus indicating the systematic track bias that is hard to be reduced by a simple ensemble with random initial conditions.

With improvement in the large-scale environment in the DAMV experiments and use of the multiple-physics LETKF algorithm to correct model errors, it is of interest to note that the systematic track bias in the CTRL forecast is generally reduced in all DAMV experiments (cf. Figs. 9c, d). The improvement is most realized for the case of Krosa, which shows indeed much reduction in the track bias at the 4-5 day lead times. For the case of Usagi, the track bias towards the south of the Tonkin Gulf is still present in the DAMV forecasts, but the DAMV ensemble can at least reduce the southward track bias in the CTRL experiments, thus improving the track forecast after 2 days (cf. Figs. 9a, c). The better performance of the DAMV ensemble forecasts is attributed to several factors including i) better sampling of the initial condition uncertainties by the LETKF algorithm, ii) use of multiple-physics option that could take into account the model errors, iii) use of different boundary conditions in our WRF-LETKF design, and iv) different large-scale environment for different 
1 ensemble members after assimilating the AMV data. It is generally difficult to conclusively isolate

2 the roles of each factor, but the study by Kieu et al. (2012) suggested that use of multiple-physics

3 options to enhance representation of model errors appears to account for more correction of track

4 biases in the WRF-LETKF system. Regardless of the individual factor, these cases demonstrate the

5 role of the LETKF algorithm in improving the track forecasts that the simple randomly perturbed

6 initial conditions in the CTRL experiments could not accomplish.

While the initial vortex inner-core structure is further improved with the use of TCR-based

8 synthetic observation in the DABV experiments, it is of interest to observe that there is no additional

9 improvement both in terms of track bias as well as the ensemble track spread in the DABV track

10 forecasts (Figs. 9c, f). Both the track forecast bias and spread are in fact very similar to those in the

11 DAMV forecasts. This similarity in the track forecasts between the DAMV and DABV experiments

12 indicates that the storm initial strength and structure have a smaller influence to subsequent track

13 forecasts once TCs become sufficiently strong, at least in these two example cycles of Krosa and

14 Usagi. In this regard, it is the improvement in the large-scale environment associated with the

15 assimilation of the AMV data that helps most improve track forecasts at the long range. The negligible

16 effect of the vortex strength and inner-core structures to the track forecasts in these two cases is not

17 however uncommon in practice, because the overall movement of TCs tends to be determined more

18 by the environmental steering flows rather than the storm initial structure, especially at longer lead

19 times (Buizza 2002; Du et al. 2012). While a vortex initial structure could have significant influence

20 to the storm development during the early development, a weak dependence of TC track on storm

21 initial strength is statistically consistent with various real-time TC forecasts using different

22 operational models as reported, e.g., in Tallapragada et al. (2015). This weak dependence is

23 particularly true for strong storms, which are embedded in already favorable conditions for them to 
reach their peak intensity, and so modification of the storm initial intensity would not change much

2 their overall movement.

In terms of intensity forecasts, Fig. 10 shows ensemble forecasts of VMAX obtained from the

4 three experiments. Despite the track improvement at the long lead times in the DAMV experiments,

5 there is insignificant improvement in intensity forecasts as compared to the CTRL. The most noticeable improvement is the more consistent weakening at 4-5 day leads time in the case of Krosa.

7 For all ensembles, both the CTRL and DAMV spreads are small during the first 12 hours, but it 8 quickly grows and covers a wide range of intensity variations after just 18 hours into integration. The 9 larger spread in the DAMV intensity forecasts of Krora appears to indicate larger uncertainties in 10 development of a weak storm that the CTRL could not obtain. Regarding the blending DABV 11 experiment, we observe that the assimilation of the TCR-synthetic data generally helps correct the 12 storm initial intensity, thus explaining a better fit of the DABV storm initial intensity with the 13 observed intensity as compared to the other two experiments. Except for the initial spin-down of the 14 model vortex due to balanced adjustment in the case of Usagi, the VMAX in all DABV forecasts tends to follow well the observed intensity, suggesting the complementary roles of the AMV and the TCR-synthetic data in improving intensity forecasts.

To more systematically evaluate the performance of different experiments, Fig. 11 shows 18 comparison of the track and intensity error statistics obtained from the CTRL, DAMV, and DABV experiments for all TC cases listed in Table 2. Here, the performance of the track forecasts is measured 20 using the standard absolute distance errors $(\mathrm{km})$ between the model forecasted location and the 21 observed location valid at the same time, which are averaged for all cycles at each corresponding lead 22 time. Likewise, the intensity errors are defined as an average of the absolute differences between the 23 model forecast and the observed VMAX at each lead time. 

overall possesses the largest track forecast errors at all lead times up to 4-5 days with the 5-day track

3

4

errors larger than $650 \mathrm{~km}$. Of the three experiments, the DAMV shows the best track forecast performance with noticeable improvement from 1-day to 3-day lead times at 95\% statistical significance. Likewise, the DABV statistics shows a signal of track improvement relative to the CTRL with most reduction in the track forecast errors between 48 and 84 hours. This track reduction in the DAMV and DABV experiments apparently demonstrates the role of the AMV data in correcting the large-scale environmental condition relative to the CTRL forecasts.

Although the reduction in the track forecast errors at the 4-day and 5-day lead times between DABV and CTRL experiment are not of statistical significance, it is interesting to note however that the DABV track forecast skill is somehow deteriorated at the 5-day lead time as compared to the CTRL forecasts, despite similar use of the AMV data as in the DAMV experiments. The negative impact of the synthetic inner-core assimilation to the track forecasts in the DABV experiments turns out to be not unique to our WRF-LETKF system. Indeed, other regional operational TC models appear to suffer from the same behaviors, whose track forecast skill is not comparable to the global GFS forecasts, despite much better initial TC structure and strength in the regional models (see, e.g., Tallapragada et al. 2014; Tong et al. 2015; Pu et al. 2016). Nonetheless, the overall positive impacts of assimilating the AMV data does suggest that inclusion of the AMV data could at least help improve the track forecast skill and offset the negative impacts of the inner-core assimilation that the CTRL forecasts could not achieve.

Unlike the track errors, intensity errors exhibit more impacts with assimilation of TCRsynthetic data. While the DABV has similar absolute and bias errors as the CTRL errors from 0-3 days, it possesses the lowest errors overall at 4-5 day lead times with the absolute errors $\sim 12 \mathrm{~m} \mathrm{~s}^{-1}$ at 
1 the 5-day lead time (Fig. 11b). In contrast, the CTRL intensity errors show a tendency of

2 underestimation of the storm intensity, even at 4-5 day lead times (Fig. 11c). The delayed benefit of

3 the TCR-synthetic data in improving intensity forecasts until after 3 days into integration is generally

4 seen for all strong intensity cycles, and it is attributed to the initial adjustment of the model vortex

5 after ingested TCR-synthetic data (see Fig. 10e). Note however that the DAMV experiments show

6 similar improvement in intensity forecasts at the 4-5 day lead times as compared to the CTRL

7 experiments, despite the simple use of the AMV data without any TCR-synthetic data. This suggests

8 that improvement in the large-scale environment plays a key role in the intensity improvement at the

9 long range, even though it would take several days to be realized, thus confirming the importance of

10 the large-scale flows in helping TCs to develop consistently. In this regard, the use of TCR-synthetic

11 data to enhance vortex inner-structure mostly aids the model storms to more quickly adapt to the

12 ambient environment in the DABV experiments. This may explain slightly larger intensity errors in

13 the DABV experiments during 2-3 days, but eventually the DABV could have the best performance

14 at the 4-5 day lead times relative to the CTRL and DAMV experiments.

While both the AMV and TCR-synthetic data show positive impacts on improvement of the track and intensity forecast performance at long ranges, it should be mentioned that use of the multiple

17 physics in the LETKF system to represent model errors is the most significant factor in increasing the ensemble spread and capturing larger uncertainties. Our sensitivity experiments in which the multiple-physics option is turned off show indeed a much reduced ensemble spread for both track and

20 intensity forecasts (not shown). On average, the ensemble spread in both the DAMV and DABV

21 forecasts with the use of multiple-physics options is larger than the CTRL ensemble spread (Fig. 12).

22 Furthermore, the ensemble track spread is generally smaller than the track forecast errors, whereas

23 the ensemble intensity spread is considerably larger than the intensity errors in all experiments. From 
1 this perspective, the different ensemble spread-error ratios between the track and intensity forecasts

2 suggests less predictability of TC intensity as compared to the TC motion. Of course, a larger

3 ensemble spread than the absolute errors in the DAMV and DABV experiments may indicate that the

4 ensemble system may not be optimal for these cases. However, the different behaviors in the

5 predictability of the track and the intensity forecasts are consistent with the fact that TC tracks are

6 mostly determined by the large-scale steering flow, whereas the TC intensity is governed more by the

$7 \quad$ TC physical processes that are not fully represented in all TC models at present.

8

\section{Conclusions}

In this study, a method to directly assimilate tropical cyclone real-time records (TCRs) into the WRF model has been presented for real-time tropical cyclone (TC) forecast applications, based on the local ensemble transform Kalman filter (LETKF) algorithm. Unlike current approaches in which either TCRs are used to generate a bogus vortex or a weak vortex is spun up to match with observed intensity, our method consists of three basic steps: 1) generate a dynamically consistent three-dimensional synthetic profile for the vortex structure based on the TCR information to enhance axisymmetric components of the TC inner-core circulation, 2) blend the synthetic profile with the CIMSS-AMV data to augment representation of the large-scale steering flows, and 3) assimilate the blended data into GFS analyses using the LETKF algorithm. Note that in our proposed approach, no vortex filtering or intensity correction is applied at any step; the synthetic-AMV data combination is directly assimilated into the GFS analysis under the assumption that the vortex location in the GFS analyses is sufficiently close to the best track location. Given current horizontal resolution of the operational NCEP GFS model of $\sim 27 \mathrm{~km}\left(0.25^{\circ}\right)$ and potentially much higher resolution in future, such an assumption about the GFS vortex location is plausible (see, e.g., Zhang and Krishnamurti 1997, 1999) and more so in future, thus allowing for a substantial simplification of the vortex 
1 initialization process in regional TC models. The benefit of assimilation of this blended TCR-AMV

2 data is that it avoids issues related to vortex initial adjustment caused by artificially filtering a GFS

3 vortex or re-inserting a bogussed vortex, while at the same time augmenting the TC inner-core

$4 \quad$ structure as expected.

Our preliminary experiments of the blending data approach with the WRF-LETKF system for

6 several selected Typhoon Usagi (2013), Krosa (2013), Nari (2013), Vongfong (2014), Rammasun

7 (2014) and Neoguri (2014) in this study showed that the proposed approach could effectively enhance

8 both the storm inner-core circulation and the ambient environment that these typhoons are embedded

9 in as compared to control experiments. Specifically, TCR-based synthetic profiles could add more

10 inner-core structure to the GFS initial vortices throughout the troposphere. Although the added inner-

11 core structure does not seem to help reduce intensity errors at 0-3 days, it could show positive impact

12 in the longer range intensity forecasts. Likewise, the AMV data enhances the environmental steering

13 flows and has significant influence to TC development at longer lead times as well. Combination of

14 the TCR and the AMV data along with use of multiple-physics options in our WRF-LETKF system

15 to represent model errors showed that both the track and intensity forecast errors can be reduced,

16 albeit the track error reduction is not of statistical significance due to a limited sample size. It was

17 noticed, however, that track forecast errors after assimilating the blended TCR profile and the AMV

18 data appear to be larger than those in the control experiments at the 5-day lead time. While we have

19 not fully understood such behaviors, the overall reduction in both the intensity bias and the spin-up

20 time of the model vortex suggests that use of the TCR-based synthetic information could at least allow

21 for model storms to quicker adjust to the ambient environment and improve the intensity forecasts at

22 longer range. Our approach will be most beneficial for future TC models that are directly initialized

23 from very high resolution global models for which storm initial location is sufficiently accurate 
1 (within a given storm location uncertainty) that there is no need to carry out artificial vortex removal

2 or insertion.

\section{Acknowledgments}

4 This research was supported NOAA HFIP (Award NA16NWS4680026) and Indiana University

5 research fund. We would like to thank two anonymous reviewers and Editor for their very helpful

6 comments and suggestions. 
2 Aberson, S. D., Aksoy, A., Sellwood, K. J., Vukicevic, T., and Zhang, X. (2015). Assimilation of

\section{References} high-resolution tropical cyclone observations with an ensemble Kalman filter using HEDAS: Evaluation of 2008-2011 HWRF forecasts. Mon. Wea. Rev., 143, 511-523.

Barker, D., and et al. (2012). The Weather Research and Forecasting Model's Community Variational/Ensemble Data Assimilation System: WRFDA. Bull. Amer. Meteor. Soc., 93, $831-843$.

Berger, H., Velden, C., Wanzong, S., and Daniels, J. (2007). Evaluation of a new quality indicator to estimate satellite-derived Atmospheric Motion Vector observation error. http://www.eumetsat.int/website/home/News/ConferencesandEvents/DAT_2042918.html. Accessed 19 March 2017.

Braun, S.A., and Tao, W.-K. (2000). Sensitivity of High-Resolution Simulations of Hurricane Bob (1991) to Planetary Boundary Layer Parameterizations. Mon. Wea. Rev., 128, 3941-3961.

Cecelski, S., Zhang, D.-L., and Miyoshi, T. (2014). Genesis of Hurricane Julia (2010) within an African Easterly wave: Developing and non-developing members from WRF-LETKF ensemble forecasts. J. Atmos. Sci., 71, 2763-2781.

Cha, D.-H, and Wang, Y. (2013). A Dynamical Initialization Scheme for Real-Time Forecasts of Tropical Cyclones Using the WRF Model. Mon. Wea. Rev., 141, 964-986.

Chou, K.-H., and Wu, C.-C. (2008). Typhoon Initialization in a Mesoscale Model-Combination of the Bogused Vortex and the Dropwindsonde Data in DOTSTAR. Mon. Wea. Rev., 136, 865879. 
1 Fujita, T. (1952). Pressure distribution within a typhoon. Geophys. Mag., 23, 437-451.

2 Hendricks, E. A., Peng, M. S., and Li, T. (2013). Evaluation of Multiple Dynamic Initialization Schemes for Tropical Cyclone Prediction. Mon. Wea. Rev., 141, 4028-4048.

4 Hendricks, E. A., Peng, M. S., Ge, X., and Li, T. (2011). Performance of a Dynamic Initialization Scheme in the Coupled Ocean-Atmosphere Mesoscale Prediction System for Tropical Cyclones (COAMPS-TC). Wea. Forecasting, 26, 650-663.

Holland, G. J. (1980., An Analytic Model of the Wind and Pressure Profiles in Hurricanes. Mon. Wea. Rev., 108, 1212-1218.

Holt, C., Szunyogh, I., Gyarmati, G., Leidner, S. M., and Hoffman, R. N. (2015). Assimilation of tropical cyclone observations: Improving the Assimilation of TCVitals, Scatterometer Winds, and Dropwindsonde Observations. Mon. Wea. Rev., 143, 3956-3980.

Holton, J. R. (2004). Introduction to Dynamic Meteorology. USA: Elsevier Academic Press.

Hunt, B. R., Kostelich, E. J., and Szunyogh, I. (2007). Efficient data assimilation for spatiotemporal chaos: A local ensemble transform Kalman Filter. Physica D, 230, 112-126.

Kang, J.-S., Kalnay, E., Liu, J., Fung, I., Miyoshi, T., and Ide, K. (2011). “Variable localization” in an ensemble Kalman filter: Application to the carbon cycle data assimilation, J. Geophys. Res., doi: 10.1029/2010JD014673

Kieu, C. Q., and Zhang, D.-L. (2009). An analytical model for the rapid intensification of tropical cyclones. Quart. J. Roy. Meteor. Soc., 135, 1336-1349. 
Kieu, C. Q., Nguyen, M. T., Hoang, T. M., Ngo-Duc, T. (2012). Sensitivity of the Track and Intensity Forecasts of Typhoon Megi (2010) to Satellite-Derived Atmospheric Motion Vectors with the Ensemble Kalman Filter. J. Atmos. Oceanic Technol., 29, 1794-1810.

Kieu, C. Q., Pham, M. T., Hoang, M. T. (2013). Application of the Multi-physics Ensemble Kalman Filter to Typhoon Forecast. Pure and Appl. Geophys., 171, 7, 1473-1497.

Kieu, C. Q., Tallapragada, V., and Hogsett, W. (2014),.Vertical structure of tropical cyclones at onset of the rapid intensification in the HWRF model. Geophys. Res. Lett., 41, 3298-3306.

Kieu, C. Q., and D.-L. Zhang, (2010). A piecewise potential vorticity inversion algorithm and its application to hurricane inner-core anomalies. J. Atmos. Sci., 67, 1745-1758.

Kurihara, Y., Bender, M. A., and Ross, R. J. (1993). An initialization scheme of hurricane models by vortex specification. Mon. Wea. Rev., 121, 2030-2045.

Liu, J., Fertig, E. J., Li, H., Kalnay, E., Hunt, B. R., Kostelich, E. J., Szunyogh, I., and Todling, R. (2008). Comparison between Local Ensemble Transform Kalman Filter and PSAS in the NASA finite volume GCM - perfect model experiments, Nonlin. Processes Geophys., 15, 645-659.

Liu, Q., Surgi, N., Lord, S., Wu, W.-S., Parrish, S., Gopalakrishnan, S., Waldrop, J., and Gamache, J. (2006). Hurricane initialization in HWRF model. Preprints, 27th Conf. on Hurricanes and Tropical Meteorology, Monterey, CA, Amer. Meteor. Soc., 8A.2. http://ams.confex.com/ams/pdfpapers/108496.pdf. Accessed 19 March 2017. 
Miyoshi, T., and Kunii, M. (2012). The Local Ensemble Transform Kalman Filter with the Weather Research and Forecasting Model: Experiments with Real Observations. Pure and Appl. Geophys., 169, 321-333.

Miyoshi, T., and Kunii, M. (2012). Using AIRS retrievals in the WRF-LETKF system to improve regional numerical weather prediction. Tellus, $64 A, 18408$.

Nguyen, H. V., and Chen, Y.-L. (2011). High-Resolution Initialization and Simulations of Typhoon Morakot (2009). Mon. Wea. Rev., 139, 1463-1491.

Nguyen, H. V., and Chen, Y.-L. (2014). Improvements to a Tropical Cyclone Initialization Scheme and Impacts on Forecasts. Mon. Wea. Rev., 142, 4340-4356.

Ott, E., and et al. (2004). A local ensemble Kalman filter for atmospheric data assimilation. Tellus, 56A, 415-428.

Ott, E., Hunt, B. R., Szunyogh, I., Corazza, M., Kalnay, E., Patil, D. J., and Yorke, J. A. (2002). Exploiting Local Low Dimensionality of the Atmospheric Dynamics for Efficient Ensemble Kalman Filtering, http://arxiv.org/abs/physics/0203058v3. Accessed 19 March 2017.

Pu, Z., and Braun, S. A. (2001). Evaluation of bogus vortex techniques with four-dimensional variational data assimilation. Mon. Wea. Rev., 129, 2023 - 2039.

Pu, Z., Zhang, S., Tong, M., and Tallapragada, V. (2016). Influence of the self-consistent regional ensemble background error covariance on hurricane inner-core data assimilation with the GSIbased hybrid system for HWRF. J. Atmos. Sci., 73, 4911-4925.

Ross, R., and Kurihara, Y. (1992). A simplified scheme to simulate asymmetries due to the beta effect in barotropic vortices. J. Atmos. Sci., 49, 1620-1628. 
1 Skamarock, W. C., Klemp, J. B., Dudhia, J., Gill, D. O., Barker, D. M., Duda, M. G., Huang, X. Y., Wang, W., and Powers, J. G. (2008)/ A description of the Advanced Research WRF Version 3. NCAR Tech. Note NCAR/TN-475+STR, 113 pp.

Snyder, C., and Zhang, F. (2003). Assimilation of simulated Doppler radar observations with an Ensemble Kalman Filter. Mon. Wea. Rev., 131, 1663-1677.

Szunyogh, I., Kostelich, E. J., Gyarmati, G., Kalnay, E., Hunt, B. R., Ott, E., Satterfield, E., and Yorke, J. A. (2008). A Local Ensemble Transform Kalman Filter data assimilation system for the NCEP global model. Tellus, 60A, 113-130.

Tallapragada, V., Kieu, C. Q., Kwon, Y., Liu, Q., Trahan, S., Zhang, Z., Tong, M., Strahl, B. (2015). Real-Time Forecasts for the 2012 North-Western Pacific Typhoon Season using the NCEP Operational HWRF. Wea. and Forecasting, 30, 1355-1373.

Tallapragada, V., Tong, M., Liu, E., Zhang, B., Wang, W., and Kieu, C. Q. (2014). Aircraft Doppler Versus Flight Level Data Impact Study using FY13 Operational HWRF with vortex initialization and One-Way Hybrid Data Assimilation Results from 2008-2012 hurricane $\begin{array}{lllll}\text { seasons. } & \text { HFIP } & \text { Telecon, } & \text { Jan } & 22,\end{array}$ http://www.hfip.org/documents/minutes/03_Vijay_HFIP_22Jan2014.pdf. Accessed 19 March 2017.

Tong, M., Tallapragada, V., Liu, E., Zhang, B., Wang, W., Kieu, C. Q., and Liu, Q. (2014). Assimilation of Aircraft Reconnaissance Observations with HWRF Hybrid Data Assimilation System, San Diego, CA, 31st Conference on Hurricanes and Tropical Meteorology, Amer.. Meteor. Soc., 14D.4. San Diego, CA. 
1 Tsyrulnikov. M.D., (2009). Is the Local Ensemble Transform Kalman Filter suitable for operational

3 Velden, C. S., Hayden, C. M., Menzel, W. P., Franklin, J. L., and Lynch, J. S. (1992). The impact of data assimilation? - COSMO Newsletter, N10, 22-36. satellite-derived winds on numerical hurricane track forecasting. Wea. Forecasting, 7, 107118.

Wang, M., Xue, M., Zhao, K., and Dong, J. (2014). Assimilation of T-TREC-Retrieved Winds from Single-Doppler Radar with an Ensemble Kalman Filter for the Forecast of Typhoon Jangmi (2008). Mon. Wea. Rev., 142, 1892-1907.

Wang, Y. (1998). On the bogusing of tropical cyclones in numerical models: The influence of vertical structure. Meteor. Atmos. Phys., 65, 153-170.

Wu, C-C., Chou, K-H., Wang, Y., and Kuo, Y-H. (2006). Tropical cyclone initialization and prediction based on four-dimensional variational data assimilation. J. Atmos. Sci., 63, 23832395.

Xiao, Q., Zou, X., and Wang, B. (2000). Initialization and Simulation of a Landfalling Hurricane Using a Variational Bogus Data Assimilation Scheme. Mon. Wea. Rev., 128, 2252-2269.

Xu, D., Liu, Z., Huang, X.-Y., Min, J., Wang, H. (2013). Impact of assimilating IASI radiance observations on forecasts of two tropical cyclones. Meteor. Atmos. Phy., 122, 1-18.

Yang, S. C., Corazza, M., Carrassi, A., Kalnay, E., and Miyoshi, T. (2009). Comparison of Local Ensemble Transform Kalman Filter, 3DVAR, and 4DVAR in a Quasigeostrophic Model. Mon. Wea. Rev., 137, 693-709. 
1 Zhang, F., and Weng, Y. (2015). Predicting Hurricane Intensity and Associated Hazards: A Five-

8 Zhang, F., Meng, Z., and Aksoy, A. (2006). Tests of an ensemble Kalman filter for mesoscale and Year Real-Time Forecast Experiment with Assimilation of Airborne Doppler Radar Observations. Bull. Amer. Meteor. Soc., 96, 25-32.

Zhang, F., Weng, Y., Gamache, J. F., and Marks, F. D. (2011). Performance of convection-permitting hurricane initialization and prediction during 2008-2010 with ensemble data assimilation of inner-core airborne Doppler radar observations. Geophys. Res. Lett., doi:10.1029/2011GL048469 regional-scale data assimilation. Part I: Perfect model experiments. Mon. Wea. Rev., 134, 722736.

Zhang, Z., and Krishnamurti, T. N. (1997). Ensemble Forecasting of Hurricane Tracks. Bull. Amer. Meteor. Soc., 78, 2785-2795.

Zhang, Z., and Krishnamurti, T. N. (1999). A Perturbation Method for Hurricane Ensemble Predictions. Mon. Wea. Rev., 127, 447-469.

Zou, X., and Xiao, Q. (2000). Studies on the Initialization and Simulation of a Mature Hurricane Using a Variational Bogus Data Assimilation Scheme. J. Atmos. Sci., 57, 836-860. 
2 Table 1. Physical parameterization schemes used in the WRF-LETKF multiple-physics ensemble configuration for all ensemble experiments

\begin{tabular}{|c|c|c|c|}
\hline Ensemble member & Cumulus parameterization & Shortwave radiation & Cloud microphysics \\
\hline 1 & Kain-Fritsch & Dudhia & Kessler \\
\hline 2 & Betts-Miller-Janjic & Goddard & Kessler \\
\hline 3 & Betts-Miller-Janjic & Dudhia & Kessler \\
\hline 4 & Kain-Fritsch & Goddard & Lin \\
\hline 5 & Kain-Fritsch & Dudhia & Lin \\
\hline 6 & Betts-Miller-Janjic & Goddard & Lin \\
\hline 7 & Betts-Miller-Janjic & Dudhia & Lin \\
\hline 8 & Kain-Fritsch & Goddard & WSM3 \\
\hline 9 & Kain-Fritsch & Dudhia & WSM3 \\
\hline 10 & Betts-Miller-Janjic & Goddard & WSM3 \\
\hline 11 & Betts-Miller-Janjic & Dudhia & WSM3 \\
\hline 12 & Kain-Fritsch & Goddard & WSM5 \\
\hline 13 & Kain-Fritsch & Dudhia & WSM5 \\
\hline 14 & Betts-Miller-Janjic & Goddard & WSM5 \\
\hline 15 & Betts-Miller-Janjic & Dudhia & WSM5 \\
\hline 16 & Kain-Fritsch & Goddard & Eta (Ferrier) \\
\hline
\end{tabular}




\begin{tabular}{|c|c|c|c|}
\hline 17 & Kain-Fritsch & Dudhia & Eta (Ferrier) \\
\hline 18 & Betts-Miller-Janjic & Goddard & Eta (Ferrier) \\
\hline 19 & Betts-Miller-Janjic & Dudhia & Eta (Ferrier) \\
\hline 20 & Kain-Fritsch & Goddard & WSM6 \\
\hline 21 & Kain-Fritsch & Dudhia & WSM6 \\
\hline
\end{tabular}

1 
Table 2. List of TCs in the northwestern Pacific basin used in ensemble experiments

\begin{tabular}{|c|c|c|c|}
\hline Storms & Start cycles & End cycles & Number of cycles \\
\hline Usagi (2013) & 2013-Sep-18 00:00 UTC & 2013-Sep-20 00:00 UTC & 4 \\
\hline Krosa (2013) & $2013-$ Oct-30 00:00 UTC & 2013-Oct-31 00:00 UTC & 3 \\
\hline Nari (2013) & 2013-Oct-10 00:00 UTC & 2013-Oct-11 00:00 UTC & 3 \\
\hline Neoguri (2014) & 2014-Jul-05 00:00 UTC & 2014-Jul-06 00:00 UTC & 2 \\
\hline Rammasun (2014) & 2014-Jul-15 00:00 UTC & 2014-Jul-15 12:00 UTC & 2 \\
\hline Vongfong (2014) & 2014-Oct-06 00:00 UTC & 2014-Oct-06 12:00 UTC & 2 \\
\hline
\end{tabular}

2

3

4 
Table 3. Descriptions of all ensemble experiments conducted with the WRF-LETKF system

\begin{tabular}{|l|l|}
\hline Convention & \\
\hline CTRL & $\begin{array}{l}\text { Control experiment, set of } 21 \text { members with different physical options, and } \\
\text { different initial conditions that are generated by adding noise to GFS analysis }\end{array}$ \\
\hline DAMV & $\begin{array}{l}\text { A set of 21 members with different physical options; initial conditions from } \\
\text { LETKF method }\end{array}$ \\
& $\begin{array}{l}\text { A set of 21 members with different physical options; the same initial conditions } \\
\text { of CTRL; having CIMSS cloud motion wind and synthetic bogus vortex } \\
\text { observation assimilation with LETKF method }\end{array}$ \\
\hline
\end{tabular}




\section{Figure captions}

3 Fig. 1 (a) GFS 5-day track forecasts and the corresponding JTWC best tracks (red line) for six TC

4

5

6

7 cases during the 2013-2014 seasons in the northwestern Pacific basin listed in Table 2; (b) an illustration of a GFS maximum 10-m wind forecast (gray columns, unit $\mathrm{ms}^{-1}$ ) and the observed intensity (black columns) for a cycle of Super Typhoon Usagi valid at 0000 UTC Sep 192013. Solid line denotes the observed minimum surface pressure (unit $\mathrm{hPa}$ ), and dashed line denotes the GFS forecast of the minimum surface pressure.

Fig. 2 Horizontal cross sections at three different levels $p=850 \mathrm{mb}$ (left panels), 500mb (middle panels) and $200 \mathrm{mb}$ (right panels) of the synthetic zonal u-wind innovations (contours, unit $\mathrm{ms}^{-}$

${ }^{1}$ ) obtained from the TCR-based synthetic profiles, and the analysis innovations obtained from the WRF-LETKF system (shaded) for (a)-(c) Typhoon Usagi valid at 0000 UTC 20 Sep 2013, and (d)-(f) Typhoon Krosa valid at 0000 UTC 31 Oct 2013. Solid contours are for the positive values, and dashed contours are for negative values. Black circle and square denote the GFS vortex center and observed center at the initial time.

Fig. 3 Similar to Fig. 2 but for meridional v-wind increments

Fig. 4 West-East vertical cross sections of the observed zonal u-wind (left panels) and v-wind innovations (right panels) obtained from the TCR-based synthetic observation (contours) and the corresponding analysis innovations (shaded) obtained from the WRF-LETKF system for (a)-(b) Typhoon Usagi valid at 0000 UTC 20 Sep 2013, and (c)-(d) Typhoon Krosa valid at 0000 UTC 31 Oct 2013.

Fig. 5 Similar to Fig. 2 but for the synthetic temperature innovations (contours, unit K), and the analysis innovations obtained from the WRF-LETKF system (shaded). 
1 Fig. 6 Similar to Fig. 2 but for the analysis specific humidity innovations (shaded, unit $\mathrm{kg} \mathrm{kg}^{-1}$ ) that are obtained from the WRF-LETKF system.

3 Fig. 7 Horizontal distributions of the CIMSS-AMV wind vector innovations (red) and the analysis wind vector innovations obtained from the WRF-LETKF system (black) at two levels $p=500 \mathrm{mb}$ (left panels) and 200mb (right panels) in the outermost domain for (a)-(b) Usagi valid at 0000 UTC 20 Sep 2013, and (c)-(d) Krosa valid at 0000 UTC 31 Oct 2013.

Fig. 8 Horizontal distributions of the observed wind vector innovations (red vectors) obtained from the DABV experiments with blending of the TCR-profiles and the AMV data, and the corresponding analysis wind vector innovations obtained from the WRF-LETKF system (black vectors) at levels $p=500 \mathrm{hPa}$ (left panels), $200 \mathrm{hPa}$ (middle panels), and zooming in within the TC region (right panels) at $990 \mathrm{hPa}$ level for (a)-(c) Typhoon Usagi valid at 0000 UTC 20 Sep 2013, and (d)-(f) Typhoon Krosa valid at 0000 UTC 31 Oct 2013. Note that only vector increments with an amplitude larger than $1 \mathrm{~m} \mathrm{~s}^{-1}$ are displayed.

Fig. 9 Ensemble track forecasts obtained from (a-b) the CTRL experiments, (c-d) the DAMV experiments, and (e-f) the DABV experiment for Typhoon Usagi valid at 0000 UTC 20 Sep 2013 (left panel) and Typhoon Krosa valid at 0000 UTC 31 Oct 2013 (right panel). Purple lines denote ensemble forecasts, the black solid line denotes the ensemble mean, and the red solid line denotes the best tracks.

Fig. 10 Time series of the maximum 10-m wind (VMAX) obtained from (a-b) the CTRL experiments, (c-d) the DAMV experiments, and (e-f) the DABV experiment for Typhoon Usagi valid at 0000 UTC 20 Sep 2013 (left panel) and Typhoon Krosa valid at 0000 UTC 31 Oct 2013 (right panel). Purple lines denote ensemble forecasts, the black solid line denotes the ensemble mean, and the red solid lines denote the observed intensity. 
1 Fig. 11 Verification of (a) the absolute track forecast errors (columns, unit km) for the CTRL (black), the DAMV (gray), and the DABV (light gray) experiments; (b)-(c) similar to (a) but for the mean absolute VMAX errors (unit, $\mathrm{m} \mathrm{s}^{-1}$ ) and the mean VMAX bias (unit $\mathrm{m} \mathrm{s}^{-1}$ ). Error bars denote the $90 \%$ confidence intervals obtained from the statistics of track and intensity forecast errors at each lead time, using the formula $\pm 1.64 \times \sigma / \sqrt{N}$, where $\sigma$ is the standard deviation, and $\mathrm{N}$ is the number of cases.

Fig. 12 (a) Time series of the ensemble track spread (bars, unit km) and track errors (solid lines, unit $\mathrm{km}$ ) that are obtained from the CTRL experiments (black bars and diamond line), the DAMV experiments (gray bars and square line), and the DABV experiments (light gray bars and triangle line); (b) similar to (a) but for the absolute VMAX spread and errors. 
Figures
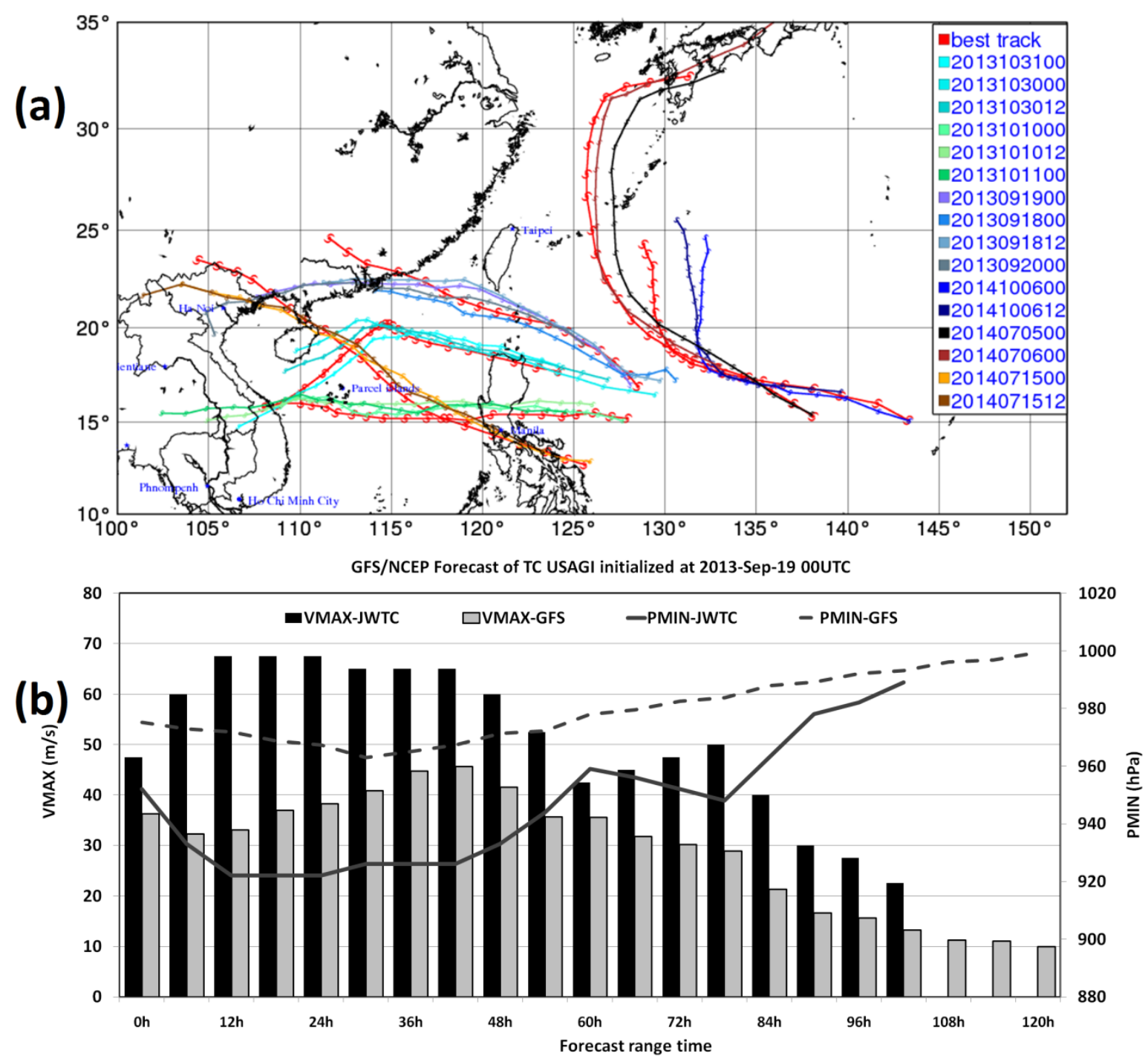

2

Fig. 1 (a) GFS 5-day track forecasts and the corresponding JTWC best tracks (red line) for six TC cases during the 2013-2014 seasons in the northwestern Pacific basin listed in Table 2; (b) an illustration of a GFS maximum 10-m wind forecast (gray columns, unit $\mathrm{ms}^{-1}$ ) and the observed intensity (black columns) for a cycle of Super Typhoon Usagi valid at 0000 UTC Sep 19 2013. Solid line denotes the observed minimum surface pressure (unit hPa), and dashed line denotes the GFS forecast of the minimum surface pressure. 

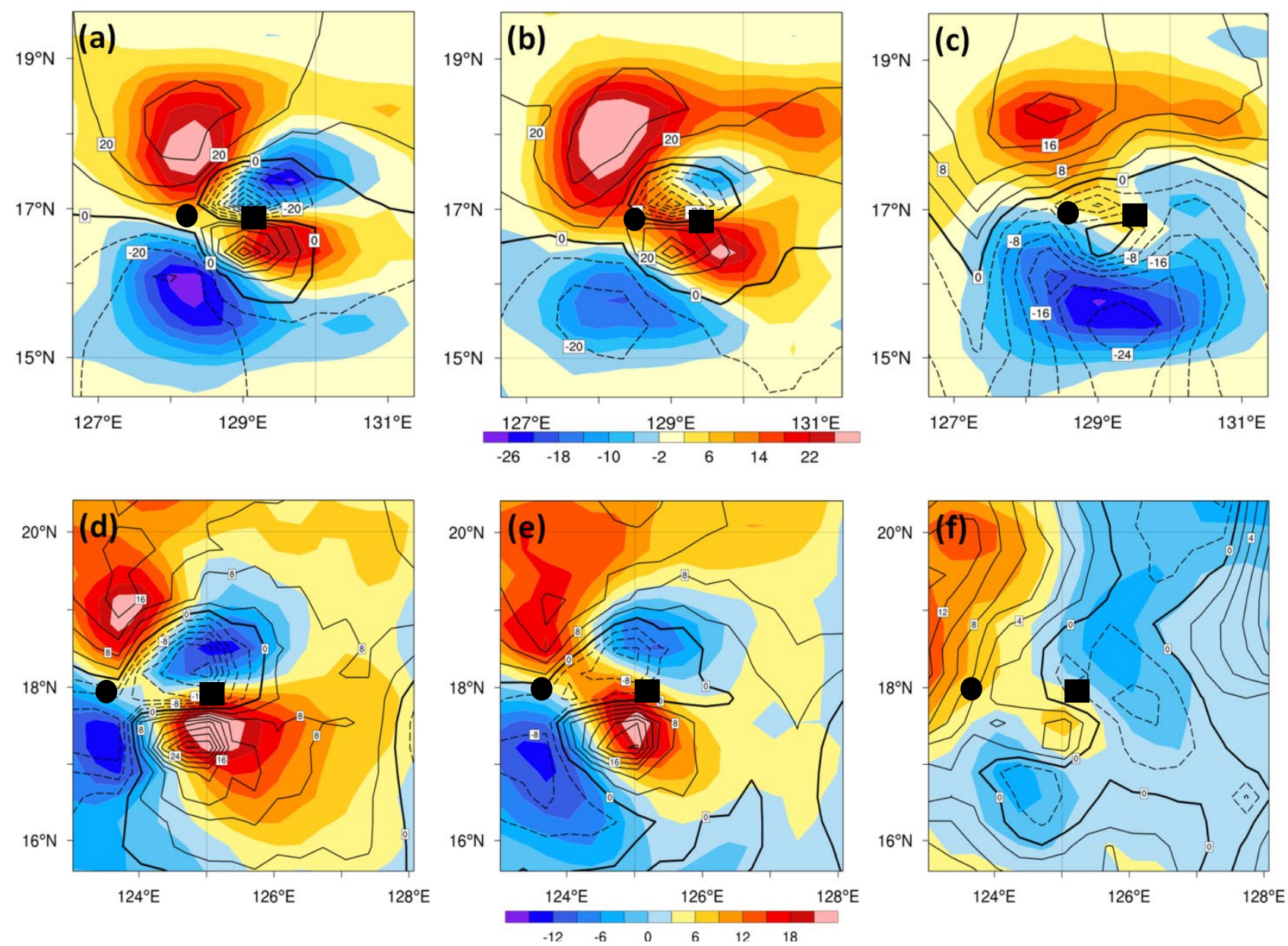

2 Fig. 2 Horizontal cross sections at three different levels $p=850 \mathrm{mb}$ (left panels), 500mb (middle

3 panels) and $200 \mathrm{mb}$ (right panels) of the synthetic zonal $\mathrm{u}$-wind innovations (contours, unit $\mathrm{ms}^{-1}$ )

4 obtained from the TCR-based synthetic profiles, and the analysis innovations obtained from the WRF-

5 LETKF system (shaded) for (a)-(c) Typhoon Usagi valid at 0000 UTC 20 Sep 2013, and (d)-(f)

6 Typhoon Krosa valid at 0000 UTC 31 Oct 2013. Solid contours are for the positive values, and dashed

7 contours are for negative values. Black circle and square denote the GFS vortex center and observed

8 center at the initial time. 

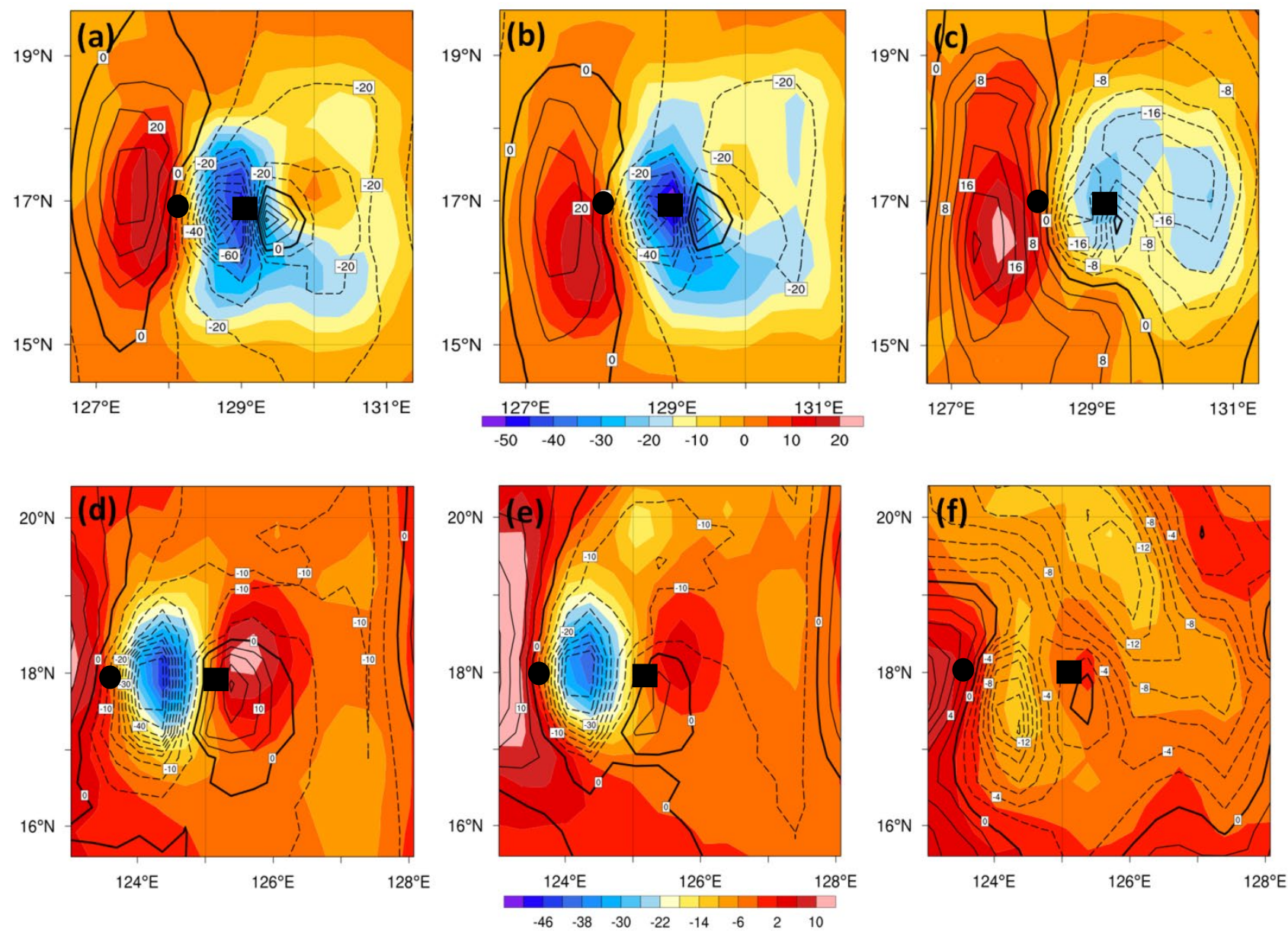

2 Fig. 3 Similar to Fig. 2 but for meridional v-wind increments 

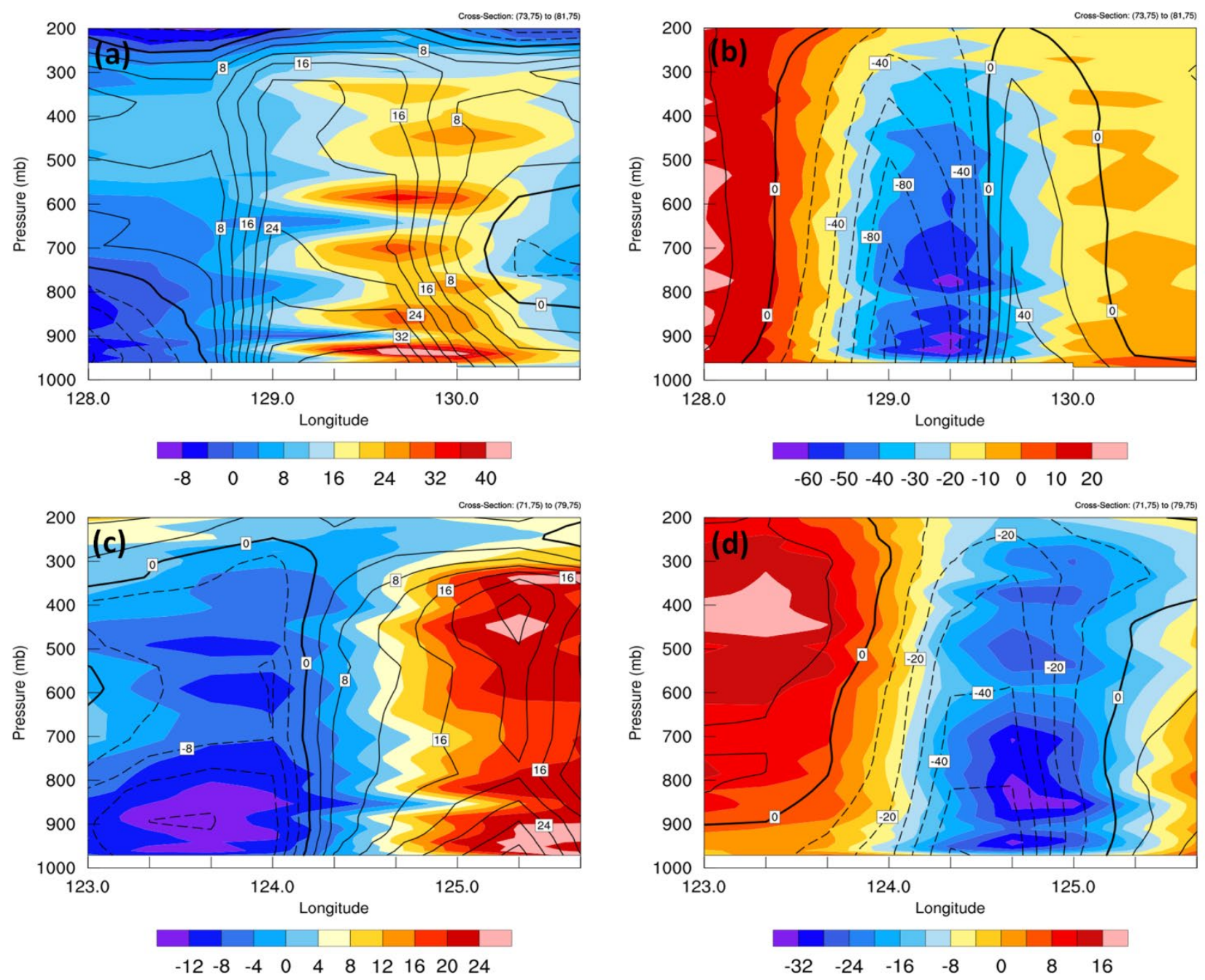

2 Fig. 4 West-East vertical cross sections of the observed zonal u-wind (left panels) and v-wind

3 innovations (right panels) obtained from the TCR-based synthetic observation (contours) and the

4 corresponding analysis innovations (shaded) obtained from the WRF-LETKF system for (a)-(b)

5 Typhoon Usagi valid at 0000 UTC 20 Sep 2013, and (c)-(d) Typhoon Krosa valid at 0000 UTC 31

6 Oct 2013. 

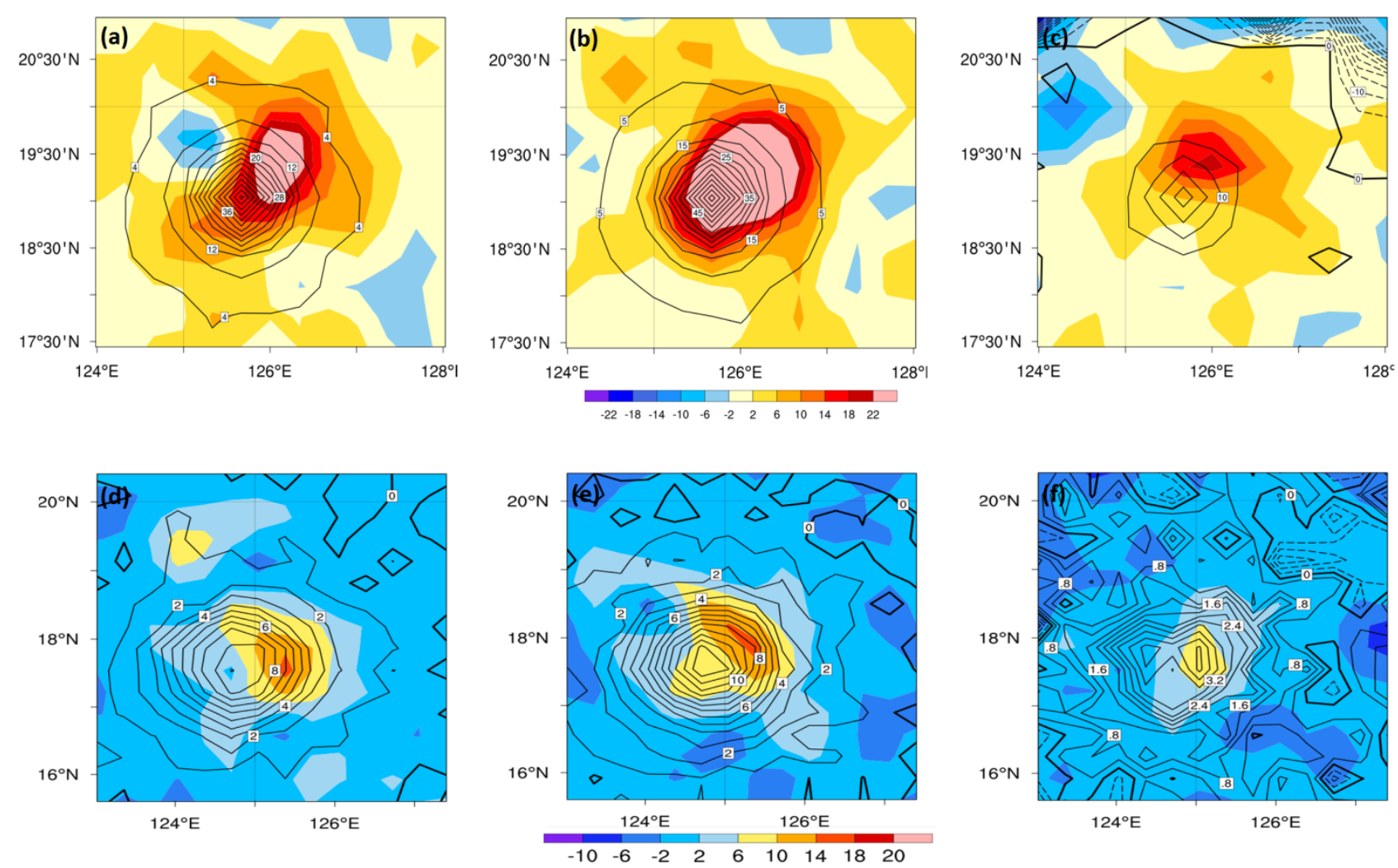

2 Fig. 5 Similar to Fig. 2 but for the synthetic temperature innovations (contours, unit K), and the 3 analysis innovations obtained from the WRF-LETKF system (shaded). 

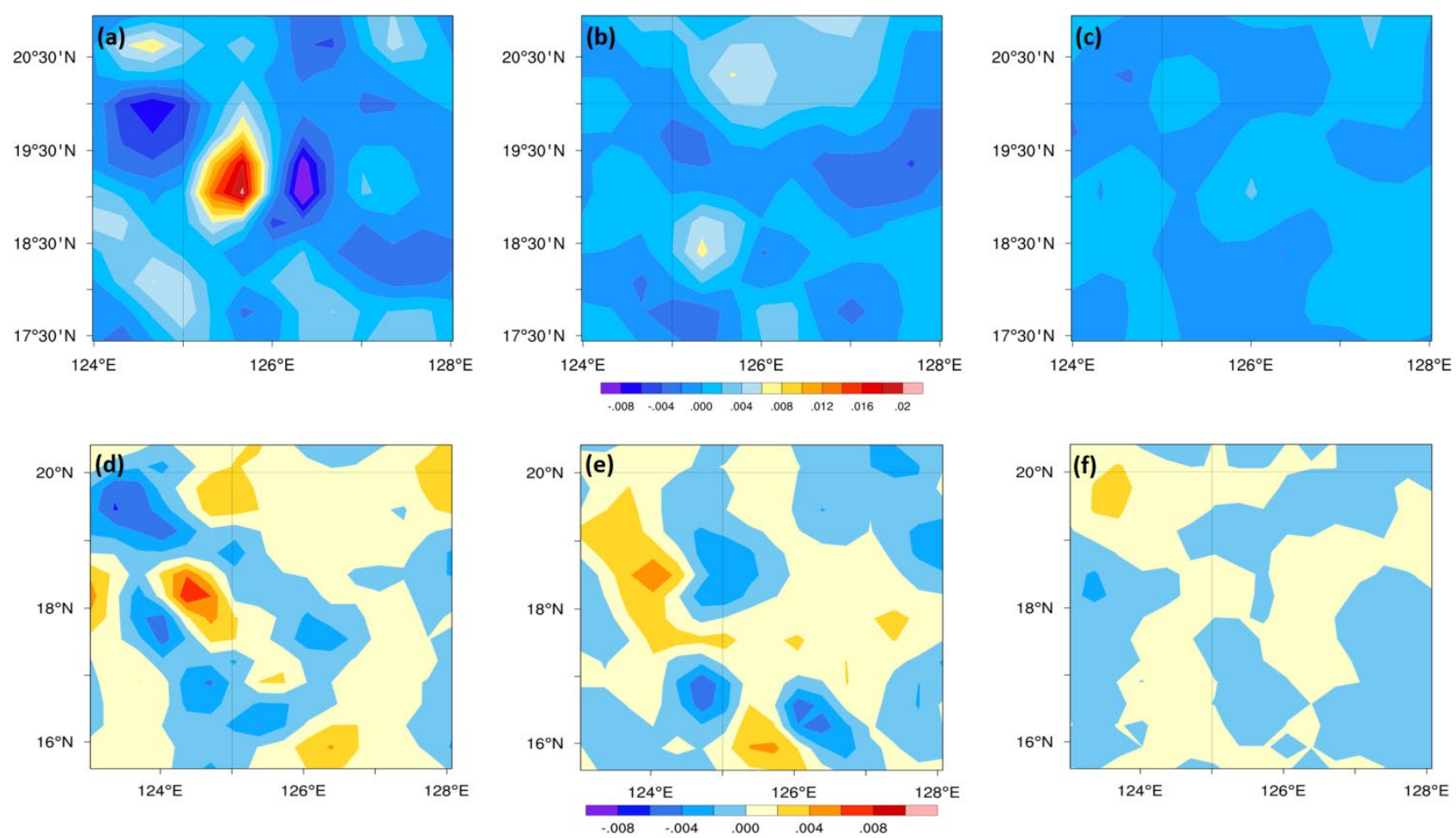

2 Fig. 6 Similar to Fig. 2 but for the analysis specific humidity innovations (shaded, unit $\mathrm{kg} \mathrm{kg}^{-1}$ ) that

3 are obtained from the WRF-LETKF system. 

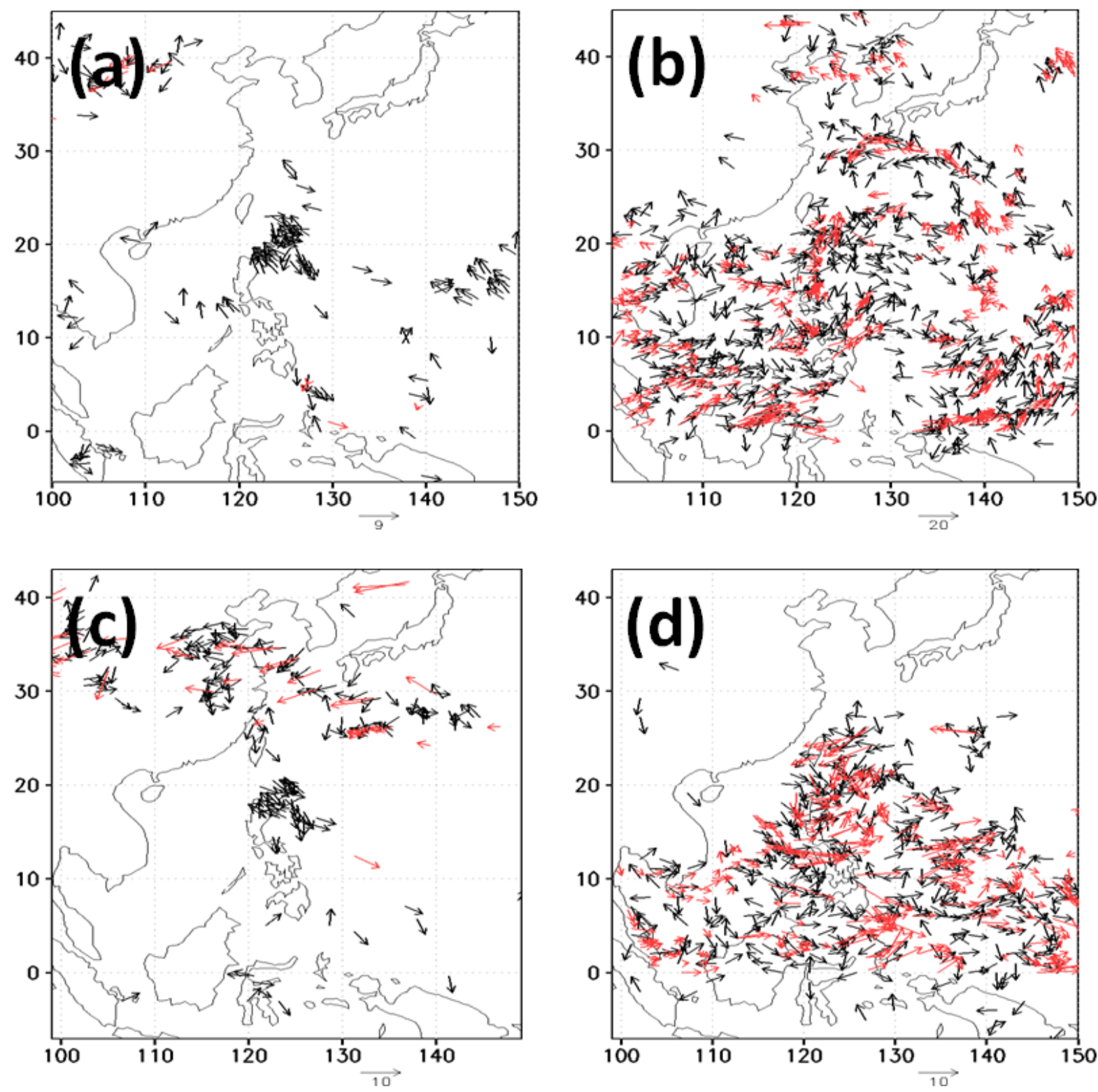

2 Fig. 7 Horizontal distributions of the CIMSS-AMV wind vector innovations (red) and the analysis

3 wind vector innovations obtained from the WRF-LETKF system (black) at two levels $p=500 \mathrm{mb}$ (left

4 panels) and 200mb (right panels) in the outermost domain for (a)-(b) Usagi valid at 0000 UTC 20 Sep

5 2013, and (c)-(d) Krosa valid at 0000 UTC 31 Oct 2013. 

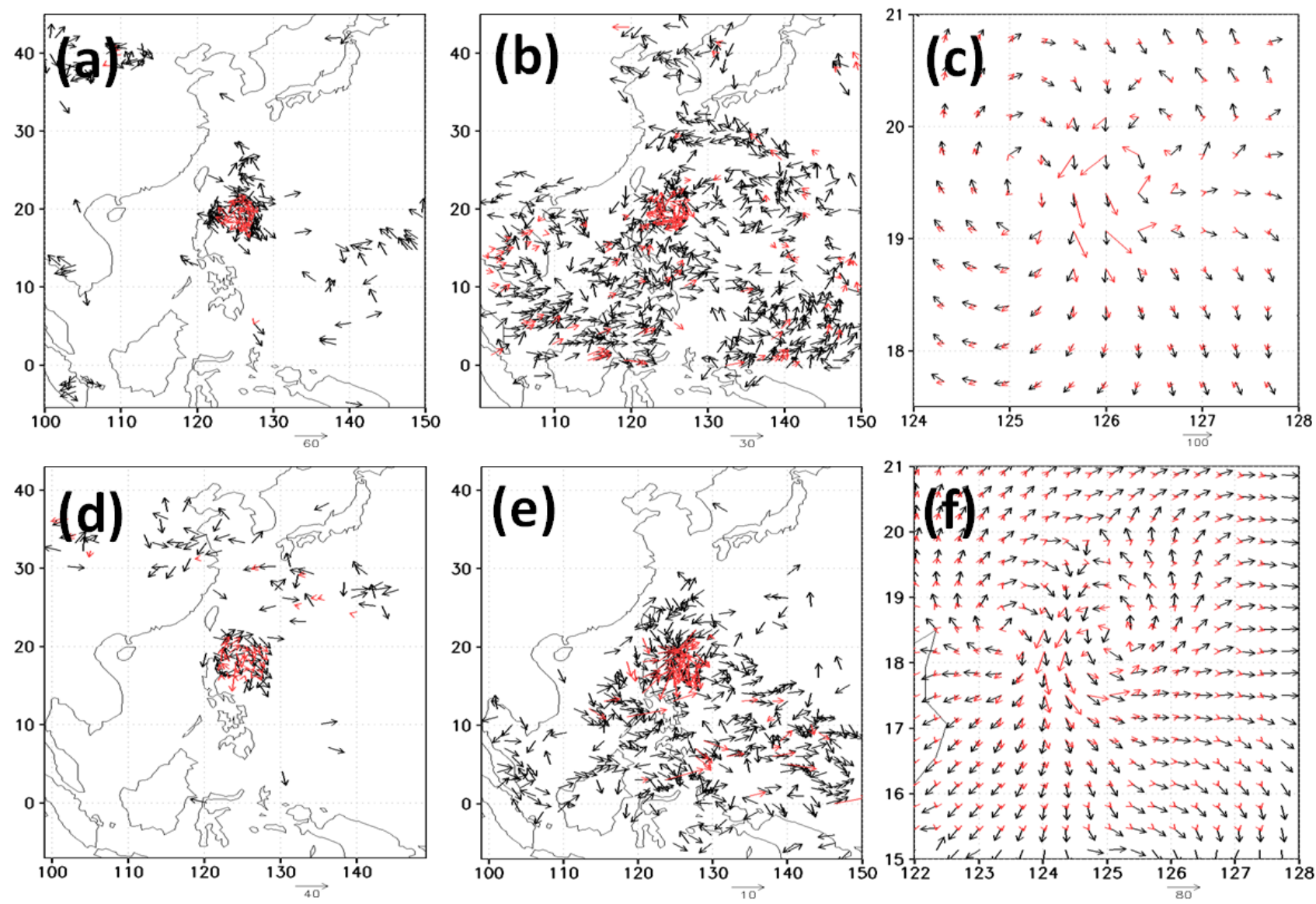

1

Fig. 8 Horizontal distributions of the observed wind vector innovations (red vectors) obtained from the DABV experiments with blending of the TCR-profiles and the AMV data, and the corresponding analysis wind vector innovations obtained from the WRF-LETKF system (black vectors) at levels $p=500 \mathrm{hPa}$ (left panels), $200 \mathrm{hPa}$ (middle panels), and zooming in within the TC region (right panels) at $990 \mathrm{hPa}$ level for (a)-(c) Typhoon Usagi valid at 0000 UTC 20 Sep 2013, and (d)-(f) Typhoon Krosa valid at 0000 UTC 31 Oct 2013. Note that only vector increments with an amplitude

$8 \quad$ larger than $1 \mathrm{~m} \mathrm{~s}^{-1}$ are displayed. 

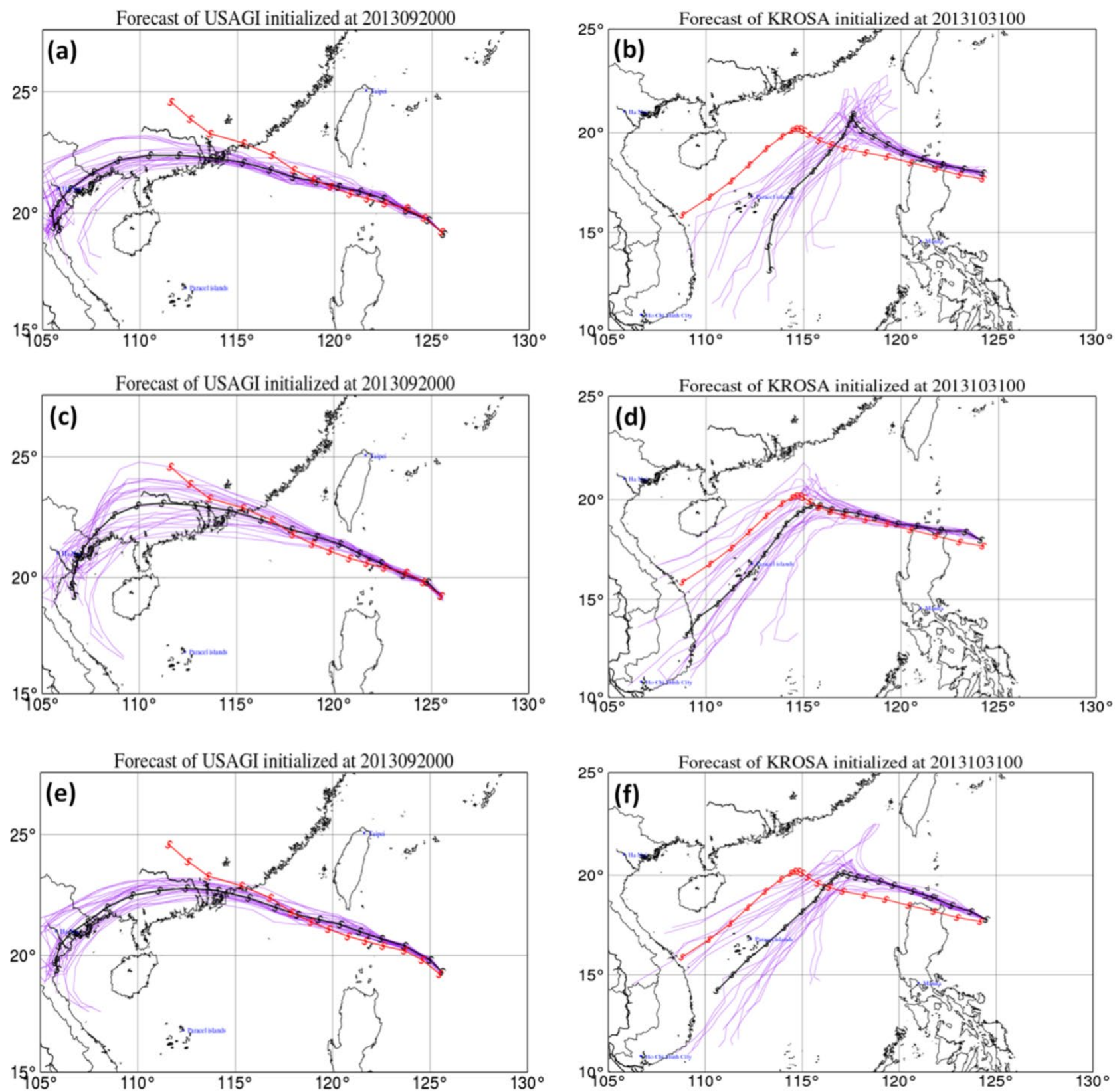

2 Fig. 9 Ensemble track forecasts obtained from (a-b) the CTRL experiments, (c-d) the DAMV

3 experiments, and (e-f) the DABV experiment for Typhoon Usagi valid at 0000 UTC 20 Sep 2013

4 (left panel) and Typhoon Krosa valid at 0000 UTC 31 Oct 2013 (right panel). Purple lines denote

5 ensemble forecasts, the black solid line denotes the ensemble mean, and the red solid line denotes the

6 best tracks. 

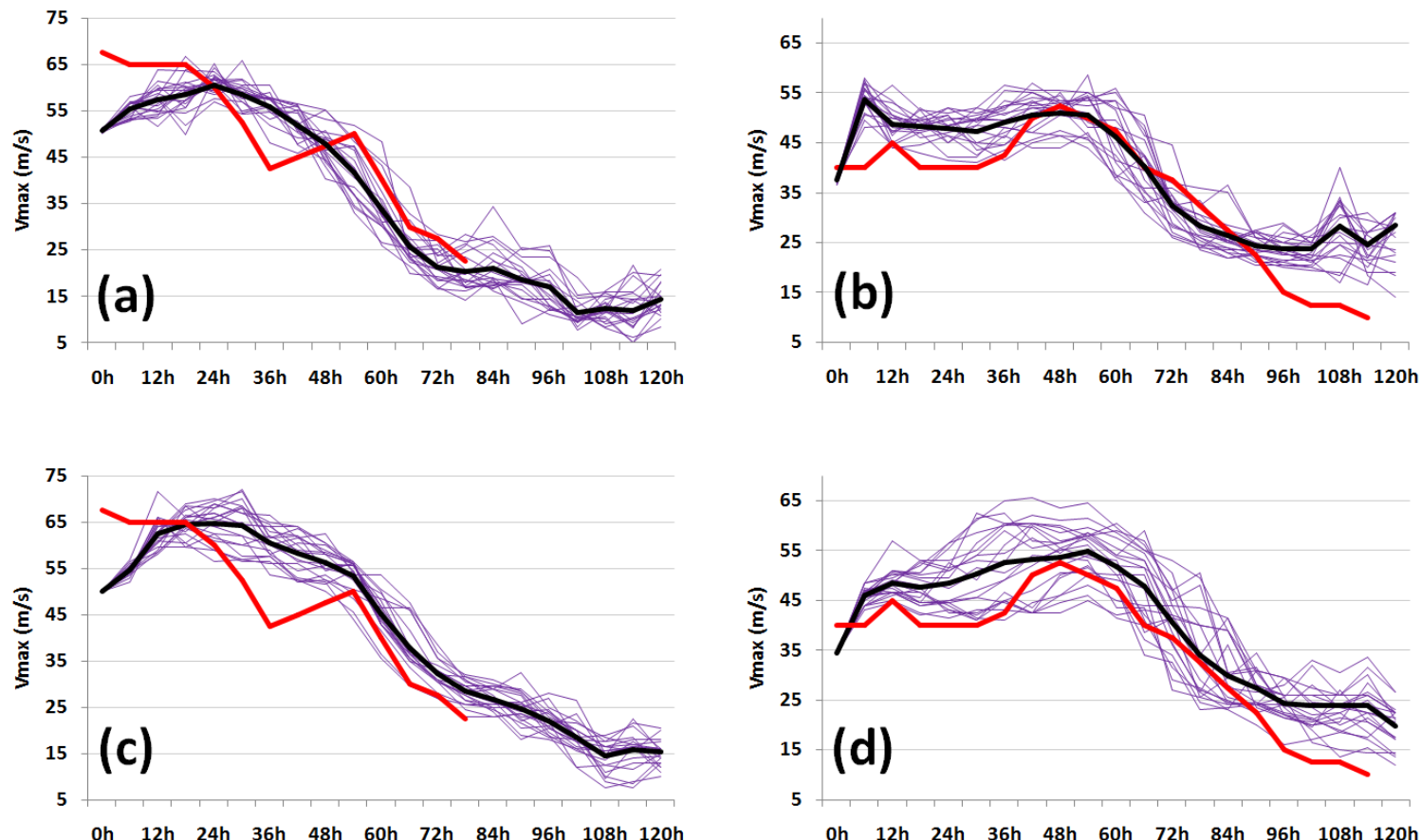

h $12 \mathrm{~h} 24 \mathrm{~h} \quad 36 \mathrm{~h} \quad 48 \mathrm{~h} \quad 60 \mathrm{~h} \quad 72 \mathrm{~h} \quad 84 \mathrm{~h} 96 \mathrm{~h} \quad 108 \mathrm{~h} 120 \mathrm{~h}$

Oh $12 \mathrm{~h} 24 \mathrm{~h} 36 \mathrm{~h} \quad 48 \mathrm{~h} \quad 60 \mathrm{~h} \quad 72 \mathrm{~h} \quad 84 \mathrm{~h} \quad 96 \mathrm{~h} \quad 108 \mathrm{~h} 120 \mathrm{~h}$
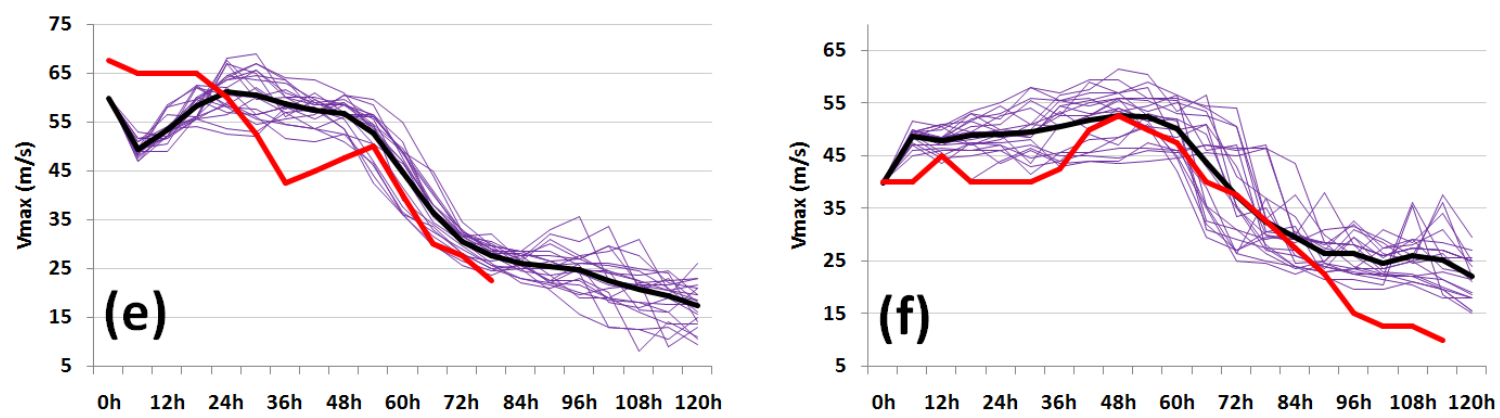

3 Fig. 10 Time series of the maximum 10-m wind (VMAX) obtained from (a-b) the CTRL experiments,

4 (c-d) the DAMV experiments, and (e-f) the DABV experiment for Typhoon Usagi valid at 0000 UTC

520 Sep 2013 (left panel) and Typhoon Krosa valid at 0000 UTC 31 Oct 2013 (right panel). Purple

6 lines denote ensemble forecasts, the black solid line denotes the ensemble mean, and the red solid

7 lines denote the observed intensity. 

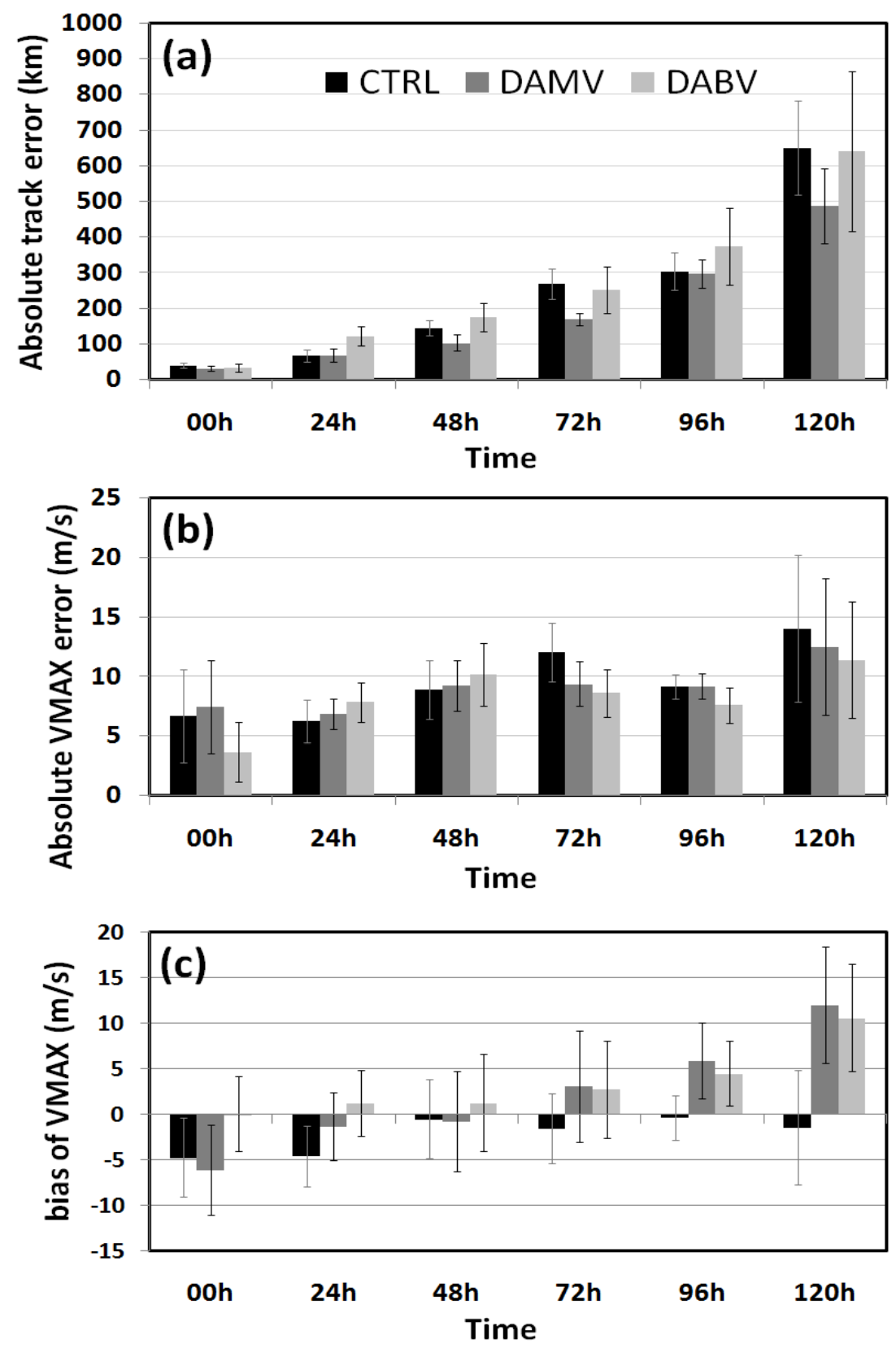

2 Fig. 11 Verification of (a) the absolute track forecast errors (columns, unit km) for the CTRL (black),

3 the DAMV (gray), and the DABV (light gray) experiments; (b)-(c) similar to (a) but for the mean

4 absolute VMAX errors (unit, $\mathrm{m} \mathrm{s}^{-1}$ ) and the mean VMAX bias (unit $\mathrm{m} \mathrm{s}^{-1}$ ). Error bars denote the $90 \%$

5 confidence intervals obtained from the statistics of track and intensity forecast errors at each lead

6 time, using the formula $\pm 1.64 \times \sigma / \sqrt{N}$, where $\sigma$ is the standard deviation, and $\mathrm{N}$ is the number of 7 cases. 

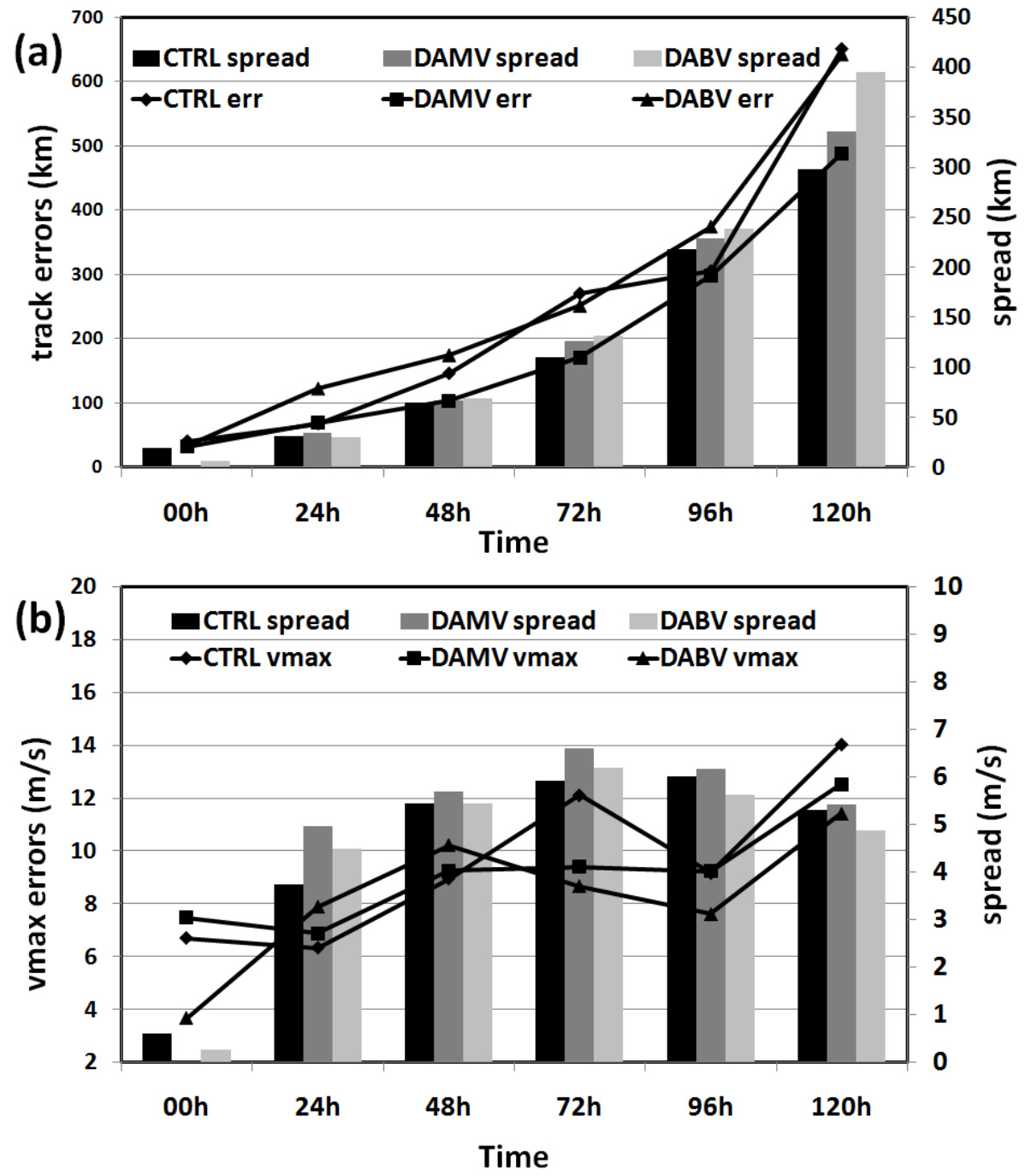

3 Fig. 12 (a) Time series of the ensemble track spread (bars, unit km) and track errors (solid lines, unit

$4 \mathrm{~km}$ ) that are obtained from the CTRL experiments (black bars and diamond line), the DAMV

5 experiments (gray bars and square line), and the DABV experiments (light gray bars and triangle

6 line); (b) similar to (a) but for the absolute VMAX spread and errors. 